\title{
ON INVARIANCE OF ORDER AND THE AREA PROPERTY FOR FINITE-TYPE ENTIRE FUNCTIONS
}

\author{
Adam Epstein and Lasse Rempe-Gillen \\ University of Warwick, Mathematics Institute \\ Coventry CV4 7AL, United Kingdom; a.l.epstein@warwick.ac.uk \\ University of Liverpool, Deptartment of Mathematical Sciences \\ Liverpool L69 7ZL, United Kingdom; l.rempe@liverpool.ac.uk
}

\begin{abstract}
Let $f: \mathbf{C} \rightarrow \mathbf{C}$ be an entire function that has only finitely many critical and asymptotic values. Up to topological equivalence, the function $f$ is determined by combinatorial information, more precisely by an infinite graph known as a line-complex. In this note, we discuss the natural question whether the order of growth of an entire function is determined by this combinatorial information. The search for conditions that imply a positive answer to this question leads us to the area property, which turns out to be related to many interesting and important questions in conformal dynamics and function theory. These include a conjecture of Eremenko and Lyubich, the measurable dynamics of entire functions, and pushforwards of quadratic differentials. We also discuss evidence that invariance of order and the area property fail in general.
\end{abstract}

\section{Introduction}

The order $\rho(f)$ of a meromorphic function $f: \mathbf{C} \rightarrow \hat{\mathbf{C}}$ is an important quantity in classical value-distribution theory [Nev53]. In the special case where $f: \mathbf{C} \rightarrow \mathbf{C}$ is an entire function, the order can be defined as

$$
\rho(f):=\limsup _{z \rightarrow \infty} \frac{\log _{+} \log _{+}|f(z)|}{\log |z|} \in[0, \infty]
$$

(where $\log _{+} x=\max (0, \log x)$ ). Any polynomial or rational function has order 0 , but there are also many transcendental entire and meromorphic functions with this property. On the other hand, the maximum modulus of an entire function can grow arbitrarily quickly; in particular, there are many functions of infinite order.

The set $S(f)$ of singular values of an entire function $f$ is the smallest closed set $S \subset \mathbf{C}$ such that

$$
f: f^{-1}(\mathbf{C} \backslash S) \rightarrow \mathbf{C} \backslash S
$$

is a covering map; equivalently, $S(f)$ is the closure of the set of all critical and asymptotic values of $f$. This set is of vital importance for both the function-theoretical and iterative study of transcendental entire (and meromorphic) functions.

It is a guiding principle of both one-dimensional holomorphic dynamics and threedimensional hyperbolic geometry that combinatorics determines geometry, under suitable finiteness assumptions. In this note, we consider a potential extension of

doi:10.5186/aasfm.2015.4034

2010 Mathematics Subject Classification: Primary 30D20; Secondary 30D05, 30D15, 30D35.

Key words: Transcendental entire function, order conjecture, area property, topological equivalence, Poincaré function, quadratic differential.

The second author was supported by EPSRC Fellowship EP/E052851/1 and a Philip Leverhulme Prize. 
this principle to value-distribution theory that was first proposed by the first author over fifteen years ago, but has not so far been discussed in print. The natural setting for our considerations is the Speiser class

$$
\mathcal{S}:=\{f: \mathbf{C} \rightarrow \mathbf{C} \text { transcendental, entire: } S(f) \text { is finite }\},
$$

which has been extensively studied both in dynamics and function theory. We shall also refer to such functions as finite type maps, in adopting terminology standard in holomorphic dynamics. We caution that the word type has an entirely different meaning in value-distribution theory. It is well-known that every function $f \in \mathcal{S}$ has $\rho(f) \geq 1 / 2$; compare [BE95, Proof of Corollary 2] or [RS99, Lemma 3.5].

To a function $f \in \mathcal{S}$, one can associate an infinite planar graph, known as the line-complex, which encodes the topological mapping behaviour of $f .{ }^{1}$ From this combinatorial data, one can reconstruct the function $f$, up to pre- and post-composition by homeomorphisms. That is, two functions $f, g \in \mathcal{S}$ have the same line-complex if and only if they are topologically equivalent in the sense of Eremenko and Lyubich:

1.1. Definition. (Topological equivalence) Two entire functions $f$ and $g$ are called topologically equivalent if there are order-preserving homeomorphisms $\varphi$ and $\psi$ such that $\psi \circ f=g \circ \varphi$.

For this reason, we shall not require the formal definition of line-complexes-for which we refer the reader to [GO08, Chapter 7]-but will instead use the notion of topological equivalence, which is easier both to define and to work with in our context. (See Section 2 for a discussion of the properties of topological equivalence.)

It is natural to ask which properties of $f$ are combinatorially determined, and, in particular, whether this is the case for the order:

1.2. Question. (Invariance of order) Let $f \in \mathcal{S}$ with $\rho(f)<\infty$, and let $g$ be topologically equivalent to $f$. Is $\rho(f)=\rho(g)$ ?

For transcendental meromorphic functions, the order is given by

$$
\rho(f)=\limsup _{r \rightarrow \infty} \frac{\log T(r, f)}{\log r}
$$

where $T(r, f)$ is the Nevanlinna characteristic of $f$. (We emphasize that knowledge of Nevanlinna theory will not be required for the remainder of the paper.) Question 1.2 is partly motivated by the fact that the answer is positive in an important, classical case: that of meromorphic functions with rational Schwarzian derivative. Indeed, in this case the order can be directly determined from the combinatorial information of the function-more precisely, $\rho(f)=\ell / 2$, where $\ell$ is the number of logarithmic singularities - and hence is indeed invariant. (See Corollary 2.5 and the discussion that precedes it.) More generally, there are a number of classical subclasses of $\mathcal{S}$ that were defined in terms of the structure of their line-complexes (e.g. maps with finitely many simply- and doubly periodic ends [Wit68], and more generally asymptotically periodic ends [GO08]). In these cases, it seems to have been implicitly understood that the order depends only on the line complex, and hence that invariance of order holds for these classes. However, the answer to Question 1.2 for general meromorphic finite-type functions is negative by work of Künzi [Kün55].

\footnotetext{
${ }^{1}$ We remark that the line-complex is uniquely defined only if one additionally fixes a suitable marking, which can be represented by a Jordan curve through the singular values.
} 
In addition to the Speiser class, the larger Eremenko-Lyubich class

$$
\mathcal{B}:=\{f: \mathbf{C} \rightarrow \mathbf{C} \text { transcendental, entire: } S(f) \text { is bounded }\}
$$

has also been studied extensively in complex dynamics. In this class, there do exist cases where the order is not invariant under topological equivalence.

1.3. Theorem. (Counterexamples in class $\mathcal{B}$ ) There exist two finite-order functions $f, g \in \mathcal{B}$ such that $f$ and $g$ are topologically equivalent, but $\rho(f) \neq \rho(g)$.

These examples arise from complex dynamics; more precisely they are given by Poincaré functions (maximally extended linearizing maps) associated to the repelling periodic cycles of polynomials. As we shall see, for a polynomial with connected Julia set, these Poincaré functions belong to the Eremenko-Lyubich class. Furthermore, an orientation-preserving topological conjugacy between polynomials induces topological equivalences between corresponding Poincaré functions (Proposition 3.2). On the other hand, in this situation the order is determined by the multiplier of the associated periodic cycle, which may change under a topological conjugacy; see Corollary 3.3.

Note that this construction cannot be extended to yield counterexamples to invariance of order in the class $\mathcal{S}$. Indeed, a Poincare function is in $\mathcal{S}$ if and only if the corresponding polynomial is postcritically finite, but postcritically finite maps are rigid by the Thurston Rigidity Theorem [DH93]. We are able to give a purely function-theoretic explanation of this phenomenon by studying an important geometric property.

The area property. The following result, which is a consequence of the wellknown Teichmüller-Wittich Theorem, will allow us to verify invariance of order for certain functions $f \in \mathcal{S}$.

1.4. Theorem. (Invariance of order and the area property) Let $f \in \mathcal{S}$, and suppose that

$$
\int_{f^{-1}(K) \backslash \mathbf{D}} \frac{\mathrm{d} x \mathrm{~d} y}{|z|^{2}}<\infty
$$

for every compact set $K \subset \mathbf{C} \backslash S(f)$. Then the order of $f$ is invariant under topological equivalence. (Here $\mathbf{D}=\{|z|<1\}$ denotes the unit disc.)

The condition (1.1) means that the cylindrical area $\operatorname{area}_{\mathrm{cyl}}\left(f^{-1}(K) \backslash \mathbf{D}\right)$-i.e. area with respect to the conformal metric $\mathrm{d} s=|\mathrm{d} z| /|z|$ on the punctured plane $\mathbf{C}^{*}$-is finite. Note that this condition makes perfect sense not just for a function $f \in \mathcal{S}$, but also for general entire functions $f$, and in particular for $f \in \mathcal{B}$ :

1.5. Definition. (The area property) We say that an entire function $f$ has the area property if (1.1) holds for every compact set $K \subset \mathbf{C} \backslash S(f)$. If $f \in \mathcal{B}$ and this property holds for every compact subset of the unbounded connected component of $\mathbf{C} \backslash S(f)$, we say that $f$ has the area property near infinity.

The area property and some variants thereof appear to be closely connected to a number of interesting questions in complex function theory and complex dynamics. In particular, a similar question was stated by Eremenko and Lyubich: 
1.6. Conjecture. (Detection of asymptotic values [EL92, p. 1009]) Suppose that $f \in \mathcal{S}$ is such that, for some $R>0$,

$$
\liminf _{r \rightarrow \infty} \frac{1}{\log r} \int_{\{z \in \mathbf{C}: 1 \leq|z| \leq r \text { and }|f(z)| \leq R\}} \frac{\mathrm{d} x \mathrm{~d} y}{|z|^{2}}>0
$$

Then $f$ has a finite asymptotic value.

In other words, suppose that $f \in \mathcal{S}$ has no finite asymptotic values. Then Conjecture 1.6 would imply that, for any compact set $K \subset \mathbf{C}$, the part of the logarithmic area of $f^{-1}(K) \backslash \mathbf{D}$ at modulus at most $r$ does not grow too quickly with $r$ (although the total logarithmic area is allowed to be infinite, in contrast to the area property). Conversely, suppose that the area property holds for $f$, and that additionally the multiplicity of the critical points of $f$ is uniformly bounded. Then the integral in (1.2) is bounded, and hence $f$ satisfies Conjecture 1.6 (see Lemma 4.4).

The area property is often easy to verify, allowing us to establish a positive answer to Question 1.2 in such cases. In particular, we can show that a Poincaré function $f$ for a polynomial $p$ with connected Julia set typically has the area property, even when invariance of order fails. Recall that, as mentioned above, such $f$ belongs to the class $\mathcal{B}$ and has finite order (see (3.2) below).

1.7. Theorem. (The area property for linearizers) Let $f \in \mathcal{B}$ be the Poincaré function associated to a repelling periodic point of a polynomial $p$ with connected Julia set. Then $f$ has the area property near infinity. Furthermore, $f$ has the area property if and only if $p$ does not have any Siegel discs. In particular, if $p$ is postcritically finite, then $f \in \mathcal{S}$ and the order of $f$ is invariant under topological equivalence.

The preceding theorem provides examples of functions in the class $\mathcal{B}$ where the area property fails. These examples rely in an essential way on the fact that the singular set of $f$ (which includes the boundary of any Siegel disc of $p$ ) disconnects the plane. So the theorem leaves open the possibility that the area property holds near infinity for all finite-order functions $f \in \mathcal{B}$, which would imply the general area property, and hence invariance of order, when $f \in \mathcal{S}$. We show that this is not the case:

1.8. Theorem. (Counterexamples to the area property near infinity) There exists a function $f \in \mathcal{B}$ of finite order such that $f$ does not have the area property near infinity.

Remark. The counterexample is constructed by precisely the same method as that used by the second author to construct a hyperbolic entire function with maximal hyperbolic dimension constructed in [Rem14]. Indeed, it can be shown that the counterexample from that paper violates the area property. For some remarks concerning connections between the area property and measurable dynamics, see Section 7 .

We consider Theorem 1.8 to provide strong evidence that invariance of order does not hold in general.

1.9. Conjecture. (Non-invariance of order) There exist entire functions $f, g \in \mathcal{S}$ such that $f$ and $g$ are topologically equivalent, but $\rho(f) \neq \rho(g)$. 
Subsequent work. While this article was being prepared, a proof of Conjecture 1.9 was announced by Chris Bishop [Bis12]. His work also includes a counterexample to Conjecture 1.6. Since his results were announced, we noticed that Poincaré functions can be used to give an alternative counterexample to the latter conjecture; we include the short argument in Proposition 4.7.

The area property is also closely connected with the question of whether the exceptional set in a certain theorem of Littlewood can be chosen to be finite. Geyer [Gey14] has independently considered Poincaré functions for polynomials with Siegel discs, as in Theorem 1.7, to construct class $\mathcal{B}$ counterexamples to this property. We refer to his paper for a discussion of the precise question.

Structure of the article. In Section 2, we introduce a number of definitions and preliminaries, and in particular recall some key facts concerning topological and quasiconformal equivalence. We also review the classical case of maps with polynomial or rational Schwarzian derivative, as studied by Nevanlinna and Elfving. In Section 3, we investigate basic properties of Poincaré functions and prove Theorem 1.3. The area property is studied in detail in Section 4, where we prove Theorem 1.4 and discuss a number of equivalent formulations of (1.1) that will be helpful in the following. We then return to Poincare functions and the proof of Theorem 1.7, which turns out to rely on a connection between the area property for the linearizer and the Poincaré series of the original polynomial. Theorem 1.8 is proved in Section 5, using a construction from [Rem14].

Finally, Section 6 discusses consequences of the area property for pushforwards of quadratic differentials, and Section 7 touches on a number of topics that are connected to our considerations, but go beyond the main scope of the article.

Basic notation. We shall assume that the reader is familiar with basic facts from complex geometry [For81], hyperbolic geometry [BM07] and the theory of quasiconformal maps [Ahl06]. In particular, we shall use the following elementary fact.

1.10. Observation. (Quasiconformal maps isotopic to a given homeomorphism) Let $\varphi: \hat{\mathbf{C}} \rightarrow \hat{\mathbf{C}}$ be an orientation-preserving homeomorphism, and let $E \subset \hat{\mathbf{C}}$ be finite.

Then there exists a quasiconformal homeomorphism $\tilde{\varphi}: \hat{\mathbf{C}} \rightarrow \hat{\mathbf{C}}$ isotopic to $\varphi$ relative $E$ and conformal near $E$. If $\# E \leq 3$, then $\tilde{\varphi}$ can be chosen to be a Möbius transformation.

If $\varphi$ is a quasiconformal map, then (following Bishop) the quasiconstant of $\varphi$ is the smallest number $K$ such that $\varphi$ is $K$-quasiconformal. Furthermore, we denote the complex dilatation of $\varphi$ by $\mu_{\varphi}$.

The Koebe Distortion Theorem [Pom92, Theorem 1.3] will also be used frequently. An important consequence of this theorem (and the Schwarz Lemma) is the standard estimate [BM07, Theorems 8.2 and 8.6] on the hyperbolic metric in a simply-connected domain: if $V \subset \mathbf{C}$ is simply-connected and $\rho_{V}$ denotes the density of the hyperbolic metric of $V$, then

$$
\frac{1}{2 \operatorname{dist}(z, \partial V)} \leq \rho_{V}(z) \leq \frac{2}{\operatorname{dist}(z, \partial V)}
$$

Throughout the paper, the complex plane, the punctured plane, the Riemann sphere and the unit disc are denoted $\mathbf{C}, \mathbf{C}^{*}, \hat{\mathbf{C}}$ and $\mathbf{D}$, respectively. 
We shall sometimes use "const" to indicate a constant in a formula. For example, $f(x) \leq$ const $\cdot|x|$ should be read as "there exists a constant $C$ such that $f(x) \leq C \cdot|x|$ ". We also write $a \asymp b$ to mean that $a$ and $b$ differ by at most a multiplicative constant; i.e. $a \leq$ const $\cdot b \leq$ const $\cdot a$. The notation $f(z) \sim g(z)($ as $z \rightarrow \infty)$ means that $\lim _{z \rightarrow \infty} f(z) / g(z)=1$.

Acknowledgments. We thank Chris Bishop, David Drasin, Lukas Geyer, Kevin Pilgrim, Stas Smirnov and, especially, Alex Eremenko, for interesting discussions, encouragement and assistance. We would also like to thank the referees for helpful comments and corrections.

\section{Maps in the Speiser and Eremenko-Lyubich class}

Singular values. Let $f: \mathbf{C} \rightarrow \mathbf{C}$ be a transcendental entire function. A point $z \in \mathbf{C}$ is called a regular value if there is an open $U \ni z$ such that $f$ maps each component of $f^{-1}(U)$ homeomorphically onto $U$. Otherwise $z$ is called a (finite) singular value, and the set of all such singular values is denoted by $S(f)$. Note that, since the set of regular values is open, this coincides with the definition of $S(f)$ given in the introduction.

Denote the sets of critical and asymptotic values of $f$ by

$$
\begin{array}{r}
C(f):=\left\{x \in \mathbf{C}: x=f(w) \text { for some } w \in \mathbf{C} \text { with } f^{\prime}(w)=0\right\} \text { and } \\
A(f):=\left\{x \in \mathbf{C}: x=\lim _{t \rightarrow 1} f(\gamma(t)) \text { for some path } \gamma:[0,1) \rightarrow \mathbf{C}\right. \text { with } \\
\qquad \gamma(t) \rightarrow \infty \text { as } t \rightarrow 1\},
\end{array}
$$

respectively. Then it follows from covering theory that

$$
S(f)=\overline{C(f) \cup A(f)} \text {. }
$$

(Clearly $C(f) \cup A(f) \subset S(f)$, and if $x$ has a neighborhood that does not intersect $C(f) \cup A(f)$, then $x$ is a regular value by the Monodromy Theorem.)

Topological and quasiconformal equivalence. Note that all three sets, $C(f)$, $A(f)$ and $S(f)$, are defined topologically, and hence are preserved by topological equivalence.

2.1. Observation. (Topological equivalence respects singular values) Suppose that $f$ and $g$ are topologically equivalent, say $\psi \circ f=g \circ \varphi$. Then $A(g)=\psi(A(f))$, $C(g)=\psi(C(f))$ and $S(g)=\psi(S(f))$.

Recall the definition of the Speiser class and the Eremenko-Lyubich class from the introduction:

$$
\begin{aligned}
& \mathcal{S}:=\{f: \mathbf{C} \rightarrow \mathbf{C} \text { transcendental, entire }: S(f) \text { is finite }\} \\
& \mathcal{B}:=\{f: \mathbf{C} \rightarrow \mathbf{C} \text { transcendental, entire }: S(f) \text { is bounded }\} .
\end{aligned}
$$

One of the key properties of the class $\mathcal{S}$ with respect to topological equivalence is that the maps $\varphi$ and $\psi$ in Definition 1.1 can always be chosen to be quasiconformal (see Proposition 2.3(d) below). For functions with infinitely many singular values, this need no longer be true, and it makes sense to introduce the following definition (see [Rem09]):

2.2. Definition (Quasiconformal equivalence). Two entire functions $f$ and $g$ are called quasiconformally equivalent if there are quasiconformal homeomorphisms 
$\varphi, \psi: \mathbf{C} \rightarrow \mathbf{C}$ such that $\psi \circ f=g \circ \varphi$. We shall refer to $\varphi$ and $\psi$ from this definition or from Definition 1.1 as witnessing homeomorphisms.

The following facts regarding topological and quasiconformal equivalence are mostly folklore (although, for the class $\mathcal{S}$, parts (a) and (d) essentially appear in [EL92, Section 3]). We provide the short proofs for completeness.

2.3. Proposition. (Properties of topological equivalence)

(a) Suppose that $f$ and $g$ are topologically equivalent, with witnessing homeomorphisms $\varphi$ and $\psi$. If a homeomorphism $\tilde{\psi}: \mathbf{C} \rightarrow \mathbf{C}$ is isotopic to $\psi$ relative $S(f)$, then there exists a homeomorphism $\tilde{\varphi}$, isotopic to $\varphi$ relative $f^{-1}(S(f))$, such that $\tilde{\varphi}$ and $\tilde{\psi}$ are also witnessing homeomorphisms for $f$ and $g$. If $\tilde{\psi}$ is quasiconformal, respectively conformal, then $\tilde{\varphi}$ is also.

(b) If $f$ and $g$ are quasiconformally equivalent and $f$ has finite positive order, then $g$ also has finite positive order. More precisely,

$$
0<\frac{1}{K} \leq \frac{\rho(g)}{\rho(f)} \leq K<\infty
$$

where $K$ is the quasiconstant of $\varphi$.

(c) Suppose that $f$ and $g$ are quasiconformally equivalent and that the witnessing homeomorphism $\varphi$ is Lipschitz at $\infty$; i.e., $|\varphi(z)| \asymp|z|$ for sufficiently large $z$. Then $\rho(f)=\rho(g)$.

(d) If $f, g \in \mathcal{S}$ are topologically equivalent, then they are quasiconformally equivalent. If $\# S(f)=2$, then $\varphi$ and $\psi$ can be chosen to be affine (and $\rho(f)=\rho(g)$ ).

Remark. It follows from the final statement in (d) that the answer to Question 1.2 is always positive when $\# S(f)=2$.

Proof. Part (a) follows by lifting the isotopy. More precisely, let $\left(\psi_{t}\right)_{t \in[0,1]}$ be an isotopy from $\psi$ to $\tilde{\psi}$. Then, on every component of $U:=f^{-1}(\mathbf{C} \backslash S(f))$, there is a unique lift $\left(\varphi_{t}\right)_{t \in[0,1]}$ of this isotopy (since $f$ is a covering map on each such component). So we have an isotopy $\varphi_{t}: U \rightarrow \varphi(U)$, and only need to show that the maps $\varphi_{t}$ extend continuously to $\partial U$ and agree with $\varphi$ there.

We may assume without loss of generality that $\psi=\varphi=$ id. Let $z_{0} \in \partial U$, and set $w_{0}:=f\left(z_{0}\right)$; we must show that $\varphi_{t}(z) \rightarrow z_{0}$ as $z \rightarrow z_{0}$ in $U$, uniformly in $t$. Let $D$ be a small simply-connected neigborhood of $z_{0}$, chosen to ensure that $f: D \rightarrow f(D)=: V$ is a proper map with no critical points except possibly at $z_{0}$. We must show that $\varphi_{t}(z) \in D$ when $z$ is sufficiently close to $z_{0}$. By continuity of $f$ and the isotopy, if $z$ is close enough to $z_{0}$, and $w:=f(z)$, then $\psi_{t}(w) \in V$ for all $t$. (Recall that $\psi_{t}\left(w_{0}\right)=w_{0}$ for all $t$.) The point $z_{t}=\varphi_{t}(z)$ is obtained by analytic continuation of $f^{-1}$ along the curve $t \mapsto \psi_{t}(w)$, which is entirely contained in $V$. Hence it follows that $z_{t} \in D$ for all $t$, as desired.

Away from the critical points of $f$, the homeomorphism $\varphi$ can be written as a composition of $\psi$ with an inverse branch of $f^{-1}$. Hence, if $\psi$ is quasiconformal resp. conformal, then $\varphi$ is also (with the same quasiconstant).

Claim (b) follows from the Hölder property of quasiconformal mappings (see e.g. [Ahl06, Theorem 2 in Chapter III]). Indeed, we have $|w|^{1 / K} / C \leq|\varphi(w)| \leq C \cdot|w|^{K}$ for a suitable constant $C$ and all sufficiently large $w$, and similarly for $\psi$. To use this 
in the formula for the order of $g$, let us write $z=\varphi(w)$. We have

$$
\begin{aligned}
\frac{\log _{+} \log _{+}|g(z)|}{\log |z|} & =\frac{\log _{+} \log _{+}|\psi(f(w))|}{\log |\varphi(w)|} \leq \frac{\log _{+} \log _{+} C \cdot|f(w)|^{K}}{\log \frac{|w|^{1 / K}}{C}} \\
& =K \cdot \frac{O(1)+\log _{+} \log _{+}|f(w)|}{O(1)+\log |w|},
\end{aligned}
$$

and hence $\rho(g) \leq K \rho(f)$. The opposite inequality follows on reversing the roles of $f$ and $g$. Item (c) is immediate from the same computation.

The final claim follows from (a) and Observation 1.10.

Maps with polynomial Schwarzian derivative. The investigations of F. and R. Nevanlinna concerning the inverse problem of value-distribution theory involved a study [Nev29, Nev32] of those transcendental meromorphic functions $f: \mathbf{C} \rightarrow \widehat{\mathbf{C}}$ whose Schwarzian derivative $\mathcal{S}_{f}=\left(\frac{f^{\prime \prime}}{f^{\prime}}\right)^{\prime}-\frac{1}{2}\left(\frac{f^{\prime \prime}}{f^{\prime}}\right)^{2}$ is a polynomial. They characterized these maps as the meromorphic functions with finitely many "logarithmic ends", or logarithmic singularities: that is, $f$ is a map of finite type, all singular values of $f$ are asymptotic (rather than critical) values, and furthermore for every $a \in S(f)$ and every sufficiently small disc $D \ni a$, the number of connected components of $f^{-1}(D)$ that are not mapped homeomorphically by $f$ is finite. Such a component is called a "logarithmic tract" and corresponds to a unique "logarithmic singularity" (see [BE95] for more details concerning the classification of inverse function singularities).

For entire functions, the condition on $\mathcal{S}_{f}$ reduces to the requirement that $f$ has polynomial nonlinearity $\mathcal{N}_{f}=\frac{f^{\prime \prime}}{f^{\prime}}$.

Slightly more generally, Elfving [Elf34] allowed finitely many critical points in addition to the finitely many logarithmic singularities, to obtain the class of transcendental meromorphic functions $f: \hat{\mathbf{C}} \rightarrow \hat{\mathbf{C}}$ with rational Schwarzian derivative. (Compare also [Eps02, Lan15].) The finite poles of $\mathcal{S}_{f}$ are precisely the critical points of $f$; in fact,

$$
\mathcal{S}_{f}(\zeta)=\frac{m}{(z-\zeta)^{2}}+O\left(\frac{1}{z-\zeta}\right)
$$

near a point $\zeta$ where $\operatorname{deg}_{\zeta} f=m$. The corresponding entire functions have rational nonlinearity, with

$$
\mathcal{N}_{f}(\zeta)=\frac{m}{z-\zeta}+O(1)
$$

near such a point $\zeta$.

A calculation of asymptotics from the defining differential equations (see pp. 298303 of [Nev53], and pp. 391-393 of [Hil97]) allows one to determine the order of these functions explicitly in terms of the degree of the Schwarzian at $\infty$. The latter, in turn, can be expressed in terms of the number of logarithmic singularities of $f$ :

2.4. Proposition. (Order of maps with rational Schwarzian) Let $f: \mathbf{C} \rightarrow \hat{\mathbf{C}}$ be a transcendental meromorphic function with rational Schwarzian derivative. Then $\rho(f)=\frac{2+\operatorname{deg}_{\infty} \mathcal{S}_{f}}{2}=\frac{\ell}{2}$, where $\ell$ is the number of logarithmic singularities of $f$. In particular, if $f$ is entire with rational nonlinearity, then $\rho(f)=1+\operatorname{deg}_{\infty} \mathcal{N}_{f}$.

2.5. Corollary. (Invariance of order for maps with rational Schwarzian) Let $f: \mathbf{C} \rightarrow \hat{\mathbf{C}}$ be a transcendental meromorphic function with rational Schwarzian derivative. Then $\rho(f)=\rho(g)$ for any topologically equivalent map $g$. 
Proof. Clearly the numbers of logarithmic singularities over infinity, logarithmic singularities over finite asymptotic values, and of critical points are preserved under topological equivalence. In particular, the function $g$ also has rational Schwarzian derivative, and hence $\rho(f)=\rho(g)$ by Proposition 2.4.

\section{Poincaré functions: Non-invariance of order in $\mathcal{B}$}

Let $h: \mathbf{C} \rightarrow \mathbf{C}$ be an entire function, and let $\zeta \in \mathbf{C}$ be a repelling fixed point of $h$. That is, $h(\zeta)=\zeta$ and $|\lambda|>1$, where $\lambda=h^{\prime}(\zeta)$ is the associated multiplier. Then there exists a unique (up to restriction) conformal map $f$, defined near 0 , such that $f(0)=\zeta, f^{\prime}(0)=1$ and

$$
f(\lambda z)=h(f(z)) .
$$

(See e.g. [Bea91, Theorem 6.3.2].) Using (3.1), we can extend $f$ to an entire function $\mathbf{C} \rightarrow \mathbf{C}$ satisfying (3.1) for all $z \in \mathbf{C}$.

3.1. Definition. (Poincaré function) The linearizing semiconjugacy $f: \mathbf{C} \rightarrow \mathbf{C}$ as above is called the Poincaré function of $f$ at $\zeta$.

If $h$ is a polynomial of degree $D$, then it is easy to verify that $f$ has finite order

$$
\rho(f)=\frac{\log D}{\log |\lambda|}
$$

(see [ES90, Formula (4)]). Moreover, the singular set $S(f)$ coincides with the postcritical set $\mathcal{P}(h)=\overline{\bigcup_{n=1}^{\infty} h^{n}(C(h))}$ of $h[\mathrm{MP} 12 \text {, Proposition 3.2 }]^{2}$. In particular, $f$ has finite type if and only if $h$ is postcritically finite, and $f$ belongs to the EremenkoLyubich class if and only if $\mathcal{P}(h)$ is bounded, which is equivalent to $J(h)$ being connected. Further function-theoretic properties of Poincaré functions have been investigated by Drasin and Okuyama [DO08].

To prove Theorem 1.3, we observe that a conjugacy between polynomials (and, in fact, entire functions) will result in the topological equivalence of their linearizers.

3.2. Proposition. (Conjugacy implies equivalence of Poincaré functions) Suppose that $h_{1}$ and $h_{2}$ are non-constant, non-linear entire functions, and that $h_{1}$ and $h_{2}$ are topologically conjugate via a homeomorphism $\psi: \mathbf{C} \rightarrow \mathbf{C}$; that is, $\psi \circ h_{1}=h_{2} \circ \psi$. Let $x_{1}$ be a repelling fixed point of $h_{1}$, set $x_{2}:=\psi\left(x_{1}\right)$, and let $f_{1}, f_{2}: \mathbf{C} \rightarrow \mathbf{C}$ be the corresponding Poincare functions of $h_{1}$ and $h_{2}$. Then there is a homeomorphism $\varphi: \mathbf{C} \rightarrow \mathbf{C}$ such that $\psi \circ f_{1}=f_{2} \circ \varphi$. If $\psi$ is quasiconformal, then $\varphi$ is also quasiconformal.

Proof. Let $\eta$ be the branch of $f_{2}^{-1}$ that takes $x_{2}$ to 0 . We first define $\varphi(z)$, provided $z$ is not a critical point of $h_{1}$. To do so, let $\alpha$ be a curve connecting 0 and $z$ and not passing through any critical point of $f_{1}$. Then $\varphi(z)$ is obtained by analytic continuation of $\eta$ along the curve $\psi \circ f_{1} \circ \alpha$.

The fact that this analytic continuation is defined, and that it is independent of the curve $\alpha$, can be seen as follows. Let $\beta_{1}$ be the concatenation of $\alpha$ with the reverse

\footnotetext{
${ }^{2}$ We remark that, in the proof of part (ii) of [MP12, Proposition 3.2], an equality is stated for the singular set $S(h \circ f)$ (using our notation above) that does not appear justified in the case where $h$ is transcendental entire. However, this equality is not, in fact, used later in the proof, so that the proposition remains correct as stated. (Also note that, both in our paper and in [MP12], the result is usually applied only when $h$ is a polynomial.)
} 
of a second curve $\hat{\alpha}$ also connecting 0 and $z$; then $\beta_{1}$ is a closed curve beginning and ending at 0 . We must show that analytic continuation of $\eta$ along $\gamma:=\psi \circ f_{1} \circ \beta_{1}$ is possible and leads to $\eta$ rather than some other branch of $f_{2}^{-1}$.

In other words, we must show that there is a curve $\beta_{2}$, beginning and ending at 0 and not passing through any critical points of $f_{2}$, such that $f_{2} \circ \beta_{2}=\gamma$. To do so, let $n$ be sufficiently large, and consider the curve

$$
\gamma^{n}:=\psi \circ f_{1} \circ \lambda_{1}^{-n} \circ \beta_{1},
$$

where $\lambda_{1}$ denotes (multiplication by) the multiplier of $h_{1}$ at $x_{1}$. For sufficiently large $n$, the curve $\gamma^{n}$ is contained in a linearizing neighborhood of $h_{2}$ around $x_{2}$, so we can set $\beta_{2}^{n}:=\eta \circ \gamma^{n}$; this is a closed curve beginning and ending at 0 . Set

$$
\beta_{2}:=\lambda_{2}^{n} \circ \beta_{2}^{n},
$$

where $\lambda_{2}$ is the multiplier of $h_{2}$ at $x_{2}$. Then $f_{2} \circ \beta_{2}=h_{2}^{n} \circ \gamma^{n}=\gamma$. Furthermore, since $\beta_{1}$ does not contain any critical points of $f_{1}$, and $\psi$ is a topological conjugacy (and hence sends critical points of $h_{1}$ to critical points of $h_{2}$ ), the curve $\gamma^{n}$ does not contain any critical points of $h_{2}^{n}$. Hence $\beta_{2}$ does not contain any critical points of $f_{2}$, as claimed.

This defines $\varphi$ with the desired property on the complement of the set of critical points of $f_{1}$. It is easy to see (e.g. by applying the same construction, but reversing the roles of $f_{1}$ and $f_{2}$ ) that $\varphi$ is a homeomorphism between the complement of the critical points of $f_{1}$ and the complement of the critical points of $f_{2}$. Since both sets are discrete, it follows that $\varphi$ extends to a homeomorphism $\varphi: \mathbf{C} \rightarrow \mathbf{C}$. (Alternatively, it is also easy to check directly that $\varphi$ extends continuously to every critical point of $f_{1}$.)

If $\psi$ is quasiconformal, then clearly $\varphi$ is quasiconformal (as it is defined as a composition of locally quasiconformal maps). In this case (which is the one we are mainly interested in), there is an alternative and shorter proof of the proposition. Indeed, we can obtain the homeomorphism $\varphi$ by solving the Beltrami equation for the pullback $f_{1}^{*} \mu_{\psi}$ of the complex dilatation of $\psi$. Since $\mu_{\psi}$ is invariant under $h_{1}$, the pullback $f_{1}^{*} \mu_{\psi}$ is invariant under $\lambda_{1}$. It follows that $\varphi$ conjugates $\lambda_{1}$ to a linear map, and hence that $g:=\psi \circ f_{1} \circ \varphi^{-1}$ semiconjugates $h_{2}$ to this linear map. Uniqueness of the Poincaré function implies $g=f_{2}$.

Proposition 3.2 implies Theorem 1.3, in the following stronger form:

3.3. Corollary. (Non-invariance of order in class $\mathcal{B}$ ) There exist two functions $f, g \in \mathcal{B}$ such that $f$ and $g$ are quasiconformally equivalent, but $\rho(f) \neq \rho(g)$.

Proof. Consider the family of quadratic polynomials

$$
p_{a}: z \mapsto a z+z^{2},
$$

with $0<|2-a|<1$. Then $p_{a}$ has a repelling fixed point of multiplier $a$ at 0 , and an attracting fixed point of multiplier $2-a$ at $1-a$. It is well-known that any two elements of this family are quasiconformally conjugate; see Proposition 23 on page 135 of [Ahl06].

Let $f_{a}$ be the Poincaré function for $p_{a}$ at 0 , and consider two polynomials in this family whose multipliers have different moduli; for example $f:=f_{3 / 2}$ and $g:=f_{4 / 3}$. By Proposition 3.2, $f$ and $g$ are quasiconformally equivalent, but $\rho(f) \neq \rho(g)$ by (3.2). 


\section{The area property}

The area property implies invariance of order. The Teichmüller-Wittich Theorem states that, if $\varphi: \mathbf{C} \rightarrow \mathbf{C}$ is quasiconformal and

$$
\lim _{R \rightarrow \infty} \int_{|z|>R}\left|\frac{\mu_{\varphi}(z)}{z^{2}}\right| \mathrm{d} x \mathrm{~d} y=0
$$

then $|\varphi(z)| \sim a \cdot|z|$ for some $a>0$. (Recall that $\mu_{\varphi}$ is the complex dilatation of $\varphi$.)

Belinski and Lehto later also showed that $\arg \varphi(z)-\arg z$ has a limit under the same assumptions, proving that $\varphi$ is in fact asymptotically conformal at infinity; i.e. $\varphi(z) \sim a z$ for some $a \in \mathbf{C}$. This is the Teichmüller-Wittich-Belinski-Lehto Theorem (see [LV65, Chapter V, §6]), sometimes also known as the TeichmüllerBelinski Theorem.

The Teichmüller-Wittich Theorem almost immediately leads to the proof of Theorem 1.4, which we shall now state somewhat more generally. In particular, we note the fact that the area property itself is preserved under suitable quasiconformal equivalence.

4.1. Proposition. (Area property, invariance of order and qc equivalence) Suppose that the entire functions $f$ and $g$ are quasiconformally equivalent, with witnessing homeomorphisms $\varphi$ and $\psi$ such that the dilatation of $\psi$ is supported on a compact subset $K \subset \mathbf{C} \backslash S(f)$. If $f$ has the area property, then $\rho(f)=\rho(g)$, and $g$ also has the area property. In particular, if $f$ belongs to the class $\mathcal{S}$ and has the area property, then $\rho(f)=\rho(g)$ for every function $g$ that is topologically equivalent to $f$.

Proof. Since $g \circ \varphi=\psi \circ f$, and $f$ and $g$ are holomorphic, the dilatation of $\varphi$ is supported on $f^{-1}(K)$. By the area property, this set has finite cylindrical area. Hence the Teichmüller-Wittich Theorem and Proposition 2.3(c) imply that indeed $\rho(f)=\rho(g)$.

If furthermore $f \in \mathcal{S}$, then by Observation 1.10 there is a quasiconformal homeomorphism $\tilde{\psi}$ that is isotopic to $\psi$ relative $S(f)$ and whose dilatation is supported away from the singular values. According to Proposition 2.3(a), there is $\tilde{\varphi}$ such that $\tilde{\psi}$ and $\tilde{\varphi}$ are witnessing homeomorphisms for the quasiconformal equivalence of $f$ and $g$. So $\rho(f)=\rho(g)$ by the first part of the current proposition, as claimed.

It remains to show that the map $g$ also has the area property. This follows from a geometric fact concerning quasiconformal mappings, which we state separately as Proposition 4.2 below for future reference.

4.2. Proposition. (Quasiconformal mappings and the area property) Suppose that $\varphi$ is a quasiconformal mapping, and that the dilatation of $\varphi$ is supported on a closed set $A$ such that $A \backslash \mathbf{D}$ has finite cylindrical area. Then $\varphi(A) \backslash \mathbf{D})$ also has finite cylindrical area.

Proof. This claim is related to the area distortion problem for quasiconformal mappings, which was solved completely by Astala [Ast94]. The estimates we require are essentially due to Gehring and Reich [GR66]. Instead of proving our claim directly using these methods, we shall formally derive it from the following result stated in Astala's article [Ast94, Lemma 3.3]. For every $K$, there is a constant $C$ with the following property. If $f: \mathbf{D} \rightarrow \mathbf{D}$ is $K$-quasiconformal with $f(0)=0$ and the dilatation of $f$ is supported on a closed subset $E \subset \mathbf{D}$, then area $(f(E)) \leq C \cdot \operatorname{area}(E)$. 
To prove our claim, let $A_{k}$, for $k \geq 0$, denote the intersection of $A$ with the annulus $\left\{2^{k}<|z|<2^{k+1}\right\}$, and set $\lambda_{k}:=\operatorname{area}_{\mathrm{cyl}}\left(A_{k}\right)$. By assumption,

$$
\sum_{k=0}^{\infty} \lambda_{k}<\infty
$$

Now consider $\tilde{A}_{k}:=\varphi\left(A_{k}\right)$ and its cylindrical area $\tilde{\lambda}_{k}$. We must show that the sequence $\tilde{\lambda}_{k}$ is also summable.

Note that the conclusion of the claim does not change under post-composition of $\varphi$ by affine functions. Hence we can assume that $\varphi(0)=0$, and (by the TeichmüllerWittich-Belinski-Lehto Theorem) that $\varphi(z) \sim z$ as $z \rightarrow \infty$.

For each $k \geq 0$, define a map $\varphi_{k}$ by

$$
\varphi_{k}(z):=\frac{\varphi\left(2^{k+1} z\right)}{2^{k+1}} .
$$

Also let $\psi_{k}$ be a Riemann map for $\varphi_{k}(\mathbf{D})$; i.e., let $\psi_{k}: \varphi_{k}(\mathbf{D}) \rightarrow \mathbf{D}$ be a conformal isomorphism with $\psi_{k}(0)=0$ and $\psi_{k}^{\prime}(0)>0$. Since $\varphi(z) \sim z$ as $z \rightarrow \infty$, we see that $\varphi_{k}$ converges uniformly to the identity. We define a quasiconformal map $f_{k}: \mathbf{D} \rightarrow \mathbf{D}$ by

$$
f_{k}:=\psi_{k} \circ \varphi_{k}
$$

Set $E_{k}:=\left\{z \in \mathbf{D}: 2^{k+1} z \in A\right\}$; i.e. $E_{k}$ is the support of the dilatation of $f_{k}$. Since cylindrical area is invariant under linear maps, we have

$$
\operatorname{area}\left(E_{k}\right) \asymp \frac{1}{2^{2(k+1)}}+\sum_{j=0}^{k} \frac{\lambda_{j}}{2^{2(k-j)}}
$$

(for a constant independent of $k$ ). For the same reason, we have

$$
\tilde{\lambda}_{k} \asymp \operatorname{area}\left(\varphi_{k+1}\left(A_{k} / 2^{k+2}\right)\right), \quad \text { and hence } \tilde{\lambda}_{k} \asymp \operatorname{area}\left(f_{k+1}\left(A_{k} / 2^{k+2}\right)\right)
$$

by the Koebe Distortion Theorem. (Observe that $A_{k} / 2^{k+2}$ is contained in the disc of radius $1 / 2$ around the origin, and hence $\varphi_{k+1}\left(A_{k} / 2^{k+2}\right)$ is well inside $\varphi_{k+1}(\mathbf{D})$. So we can indeed apply the Distortion Theorem to the map $\psi_{k+1}^{-1}$ on $f_{k+1}\left(A_{k} / 2^{k+2}\right)$.) As $A_{k} / 2^{k+2} \subset E_{k+1}$ by definition, it follows that

$$
\tilde{\lambda}_{k} \leq \text { const } \cdot \operatorname{area}\left(f_{k+1}\left(E_{k+1}\right)\right) .
$$

By Astala's result stated above, we thus see that

$$
\tilde{\lambda}_{k} \leq \mathrm{const} \cdot\left(\frac{1}{2^{2(k+2)}}+\sum_{j=0}^{k+1} \frac{\lambda_{j}}{2^{2(k+1-j)}}\right)
$$

Hence

$$
\sum_{k=0}^{\infty} \tilde{\lambda}_{k} \leq \mathrm{const} \cdot\left(1+\sum_{k=0}^{\infty} \sum_{j=0}^{k+1} \frac{\lambda_{j}}{2^{2(k+1-j)}}\right) \leq \mathrm{const} \cdot\left(1+\sum_{j=0}^{\infty} \lambda_{j} \sum_{m=0}^{\infty} \frac{1}{4^{m}}\right)<\infty .
$$

Remark. Astala states his lemma for closed subsets of the disc, but appears to prove it only when the set $E$ is compact. Since his estimates depend only on $K$, the version for closed subsets can be reduced to the compact one. Alternatively, for each $k$ we can solve the Beltrami equation to obtain a map $\sigma_{k}$ whose dilatation agrees with that of $\varphi$ for $|z|<2^{k}$ and is zero otherwise. It is easy to see that $\sigma_{k} \rightarrow \varphi$ (since 
the corresponding dilatations converge almost everywhere), and area $\left(\sigma_{k}\left(A_{j}\right)\right) \rightarrow \tilde{\lambda}_{j}$ as $k \rightarrow \infty$ for all $j$. We can then easily obtain the desired conclusion by applying the proof as above, replacing $\varphi$ by $\sigma_{k}$ in the definition of $f_{k}$; then the dilatation of $f_{k}$ has compact support. Using the fact that all estimates are uniform, we easily obtain the desired conclusion.

Some equivalent formulations of the area property. We now discuss some formulations of the area property that are easy to verify. We begin with an infinitesimal version:

4.3. Proposition (Infinitesimal area property). A transcendental entire function $f$ has the area property if and only if

$$
\sum_{z \in f^{-1}(w) \backslash \mathbf{D}} \frac{1}{|z|^{2}\left|f^{\prime}(z)\right|^{2}}<\infty
$$

for all $w \in \mathbf{C} \backslash S(f)$. Furthermore, if (4.1) holds for some $w_{0} \in \mathbf{C} \backslash S(f)$, then it also holds for all $w$ that belong to the same component of $\mathbf{C} \backslash S(f)$ as $w_{0}$.

Proof. Let $w \in S(f)$, and let $D=D_{w} \subset \mathbf{C} \backslash S(f)$ be a closed topological disc whose interior contains $w$. Let $\tilde{D} \subset \mathbf{C} \backslash S(f)$ be a slightly larger simply-connected domain with $D \subset \tilde{D}$.

Let $z \in f^{-1}(w) \backslash \mathbf{D}$, and let $V_{z}$ be the component of $f^{-1}(D)$ containing $z$. If $\tilde{V}$ is the component of $f^{-1}(\tilde{D})$ containing $V_{z}$, then $f: \tilde{V} \rightarrow \tilde{D}$ is a conformal isomorphism, and if $D_{w}$ was chosen sufficiently small, then $V$ does not intersect the disc of radius $1 / 2$ around the origin. It follows that

$$
\min _{\zeta \in V} \frac{1}{|\zeta|^{2}\left|f^{\prime}(\zeta)\right|^{2}} \asymp \operatorname{area}_{\mathrm{cyl}}\left(V_{z}\right) \asymp \max _{\zeta \in V} \frac{1}{|\zeta|^{2}\left|f^{\prime}(\zeta)\right|^{2}}
$$

by the Koebe Distortion Theorem. In particular, the area property implies (4.1).

For the "if" direction, suppose that (4.1) holds and $K \subset S(f)$ is an arbitrary compact set. Then we can cover $K$ by finitely many discs $D_{w_{1}}, \ldots, D_{w_{k}}$ as above, and it follows that $\operatorname{area}_{\text {cyl }}\left(f^{-1}(K) \backslash \mathbf{D}\right)<\infty$. Furthermore, (4.2) shows that (4.1) is an open and closed property, and hence the final claim follows.

The preceding proof relies crucially on the Koebe Distortion Theorem. It is well-known that area distortion theorems hold also for branched covering maps of bounded degree. This allows us to deduce that the area property will hold not only near regular values (as in Definition 1.5), but also near non-asymptotic critical values for which the degree of the critical points is bounded. In particular, this justifies the remark after Conjecture 1.6.

4.4. Lemma. (Bounded criticality) Let $f$ be a transcendental entire function. Let $s$ be an isolated point of $S(f)$ that is not an asymptotic value and such that the local degree of $f$ near any preimage of $s$ is uniformly bounded by a constant $\Delta$. Let $D$ be a round disc around $s$ such that $\bar{D} \cap S(f)=\{s\}$. If the condition (4.1) holds for all $z \in D^{*}:=D \backslash\{s\}$, then $f^{-1}(D) \backslash \mathbf{D}$ has finite cylindrical area.

Proof. Let $\tilde{D}$ be a slightly larger round disc around $D$ whose closure still does not intersect the singular set except in $s$. By postcomposing with an affine map, we may assume for convenience that $\tilde{D}=\mathbf{D}$. 
Let $\tilde{V}$ be a component of $f^{-1}(\tilde{D})$ that does not intersect D. The assumptions imply that $\tilde{V}$ is simply-connected and contains a unique preimage $c$ of $s$, of some degree $d \in\{1, \ldots, \Delta\}$. Let $\varphi: \mathbf{D} \rightarrow \tilde{V}$ be a conformal isomorphism with $\varphi(0)=c$. It follows that $f(\varphi(z))=\vartheta \cdot z^{d}$ for some $\vartheta \in \mathbf{C}$ with $|\vartheta|=1$ and all $z \in \mathbf{D}$; by precomposing $\varphi$ with a rotation we can assume that $\vartheta=1$.

Let $r<1$ denote the radius of $D$, so that $D=B_{r}(0)$ (where we use the standard notation for Euclidean balls). Hence $V_{c}:=\varphi\left(B_{r^{1 / d}}(0)\right)$ is the component of $f^{-1}(D)$ containing $c$. We may assume without loss of generality that $r>1 / 2$. Define $z:=\varphi\left(2^{-1 / d}\right) \in V_{c}$; then $f(z)=1 / 2$. We have $\left|f^{\prime}(z)\right| \cdot\left|\varphi^{\prime}\left(2^{-1 / d}\right)\right|=d \cdot 2^{-(d-1) / d}$ by the functional equation. By the Koebe Distortion Theorem, it follows that

$$
\operatorname{area}_{\mathrm{cyl}}\left(V_{c}\right)=\operatorname{area}_{\mathrm{cyl}}\left(\varphi\left(B_{r^{1 / d}}\right)\right) \asymp \frac{\left|\varphi^{\prime}\left(2^{-1 / d}\right)\right|}{\left|\varphi\left(2^{-1 / d}\right)\right|}=\frac{d}{2^{\frac{d-1}{d}} \cdot|z| \cdot\left|f^{\prime}(z)\right|} \asymp \frac{1}{|z| \cdot\left|f^{\prime}(z)\right|} .
$$

(Here the constants depend on $\Delta$ and $r$, but not otherwise on $f$. In particular, they are independent of the choice of $\tilde{V}$.)

So the total logarithmic area of all of these preimages $V_{c}$ is bounded in terms of the sum (4.1) for $w=1 / 2$. It remains to show that the part of $f^{-1}(D) \backslash \mathbf{D}$ that is contained in preimage components of $\tilde{D}$ that do intersect the unit disc has finite area. But each such component is bounded, and hence has finite area. Furthermore, by the local mapping properties of holomorphic functions, the number of components of $f^{-1}(\tilde{D})$ is locally finite, and hence there are only finitely many components that intersect $\overline{\mathbf{D}}$. The claim follows.

There are various other ways to reformulate the area property. For example, since $f$ is a covering map on every component of $f^{-1}(\mathbf{C} \backslash S(f))$, the derivative $f^{\prime}(z)$ can be expressed in terms of the hyperbolic metric of $f^{-1}(\mathbf{C} \backslash S(f))$. The hyperbolic metric of simply-connected domains is particularly easy to estimate in terms of the distance to the boundary, and hence we obtain the following.

4.5. Proposition. (Distances and the area property) Let $f$ be a transcendental entire function, and let $w \in \mathbf{C} \backslash S(f)$. Let $K \subset \mathbf{C} \backslash\{w\}$ be a closed connected set with $S(f) \subset K$ and $\# K>1$. Then (4.1) holds if and only if

$$
\sum_{z \in f^{-1}(w) \backslash \mathbf{D}} \frac{\operatorname{dist}\left(z, f^{-1}(K)\right)^{2}}{|z|^{2}}<\infty .
$$

Proof. Let $z \in f^{-1}(w)$, let $W$ be the component of $\mathbf{C} \backslash K$ containing $w$ and let $V$ be the component of $f^{-1}(W)$ containing $z$. Then $f: V \rightarrow W$ is a holomorphic covering map. If $\rho_{V}$ and $\rho_{W}$ denote the densities of the hyperbolic metrics of $V$ and $W$, we thus have

$$
\left|f^{\prime}(z)\right|=\rho_{V}(z) / \rho_{W}(w) .
$$

The domain $W$ is either simply-connected or conformally equivalent to the punctured unit disc (if $K$ is bounded and $W$ is the unbounded connected component of $\mathbf{C} \backslash K)$. The only covering spaces of the punctured disc are given by the universal covering (via the exponential map) and the punctured disc (via $z \mapsto z^{d}, d \geq 1$ ). The latter case cannot occur in our setting, since $f$ is transcendental; so we see that $V$ is simply-connected. The claim now follows from (4.3) and the standard estimate (1.3). 
A return to Poincaré functions. We now study the area property for Poincaré functions, proving Theorem 1.7.

4.6. Theorem. (Area property for linearizers) Let $p$ be a polynomial of degree at least 2 with a repelling fixed point at 0 , and let $f: \mathbf{C} \rightarrow \mathbf{C}$ be the Poincaré function for this fixed point. Let $w \in \mathbf{C} \backslash \mathcal{P}(p)=\mathbf{C} \backslash S(f)$. Then (4.1) holds for $w$ if and only if $w$ does not belong to a Siegel disc of $p$.

Proof. It suffices to prove the theorem for $w \in F(p)$. Indeed, if $w \in J(p)$, then we can let $w^{\prime}$ be a point in the basin of infinity of $p$ that belongs to the same component of $\mathbf{C} \backslash S(f)$ as $w$. By Proposition 4.3, property (4.1) holds for $w^{\prime}$ if and only if it holds for $w$.

Let $\eta<1$ be small enough so that $f$ is injective on a neighbourhood of the closed disc of radius $\eta$ around 0 . We define

$$
A:=\{z \in \mathbf{C}: \eta /|\lambda|<|z|<\eta\},
$$

where $\lambda=p^{\prime}(0)$. For simplicity, we may assume that $\eta$ is chosen such that $f(\partial A)$ does not intersect the backwards orbit $\bigcup_{n=0}^{\infty} p^{-n}(w)$.

Suppose that $z \in f^{-1}(w) \backslash \overline{\mathbf{D}}$, and let $n \geq 1$ be minimal such that $|\lambda|^{n} \geq|z| / \eta$. Set $\tilde{z}:=z / \lambda^{n}$ and $\tilde{w}:=f(\tilde{z})$. By the functional relation $f \circ \lambda=p \circ f$, we have

$$
\begin{aligned}
p^{n}(f(\tilde{z})) & =f\left(\lambda^{n} \tilde{z}\right)=f(z)=w \text { and } \\
|z| \cdot\left|f^{\prime}(z)\right| & =|z| \cdot \frac{\left|\left(p^{n}\right)^{\prime}(\tilde{w})\right| \cdot\left|f^{\prime}(\tilde{z})\right|}{\lambda^{n}}=|\tilde{z}| \cdot\left|f^{\prime}(\tilde{z})\right| \cdot\left|\left(p^{n}\right)^{\prime}(\tilde{w})\right| .
\end{aligned}
$$

In particular, by our assumption on $\eta$, we have $\tilde{z} \in A$, and hence the numbers $|\tilde{z}|$ and $\left|f^{\prime}(\tilde{z})\right|$ are uniformly bounded away from 0 and $\infty$. Furthermore, for every $n \geq 1$, the correspondence between $z$ and $\tilde{w}$ defines a bijection between the points of $f^{-1}(w)$ of modulus between $\left|\lambda^{n-1}\right|$ and $\left|\lambda^{n}\right|$ and the intersection $p^{-n}(w) \cap f(A)$. So, for $N \geq 1$,

$$
\sum_{z \in f^{-1}(w), 1 \leq|z| \leq \lambda^{N}} \frac{1}{|z|^{2}\left|f^{\prime}(z)\right|^{2}} \asymp \sum_{n=1}^{N} \sum_{\tilde{w} \in f(A) \cap p^{-n}(w)} \frac{1}{\left|\left(p^{n}\right)^{\prime}(\tilde{w})\right|^{2}} .
$$

If $w$ does not belong to a Siegel disc, then it lies in the basin of infinity of $p$, an atttracting or parabolic basin, or a Fatou component that is not periodic. In each case, we can find a small disc $D$ around $w$ such that $p^{-n}(D) \cap D=\emptyset$ for all $n \geq 1$. So the sum

$$
\sum_{n=0}^{\infty} \sum_{\tilde{w} \in p^{-n}(w)} \frac{1}{\left(p^{n}\right)^{\sharp}(\tilde{w})^{2}}
$$

(the Poincaré series at exponent 2) is comparable to the spherical area of the backward orbit of the disc $D$ under $p$, and hence finite. (Here we use $\left(p^{n}\right)^{\sharp}$ to denote the derivative of $p^{n}$ as measured with respect to the spherical metric, both in the range and in the domain.) Since the spherical metric and the Euclidean metric are comparable on the bounded set $f(A)$, we see from (4.4) that $f$ satisfies (4.1).

On the other hand, suppose that $w$ belongs to a Siegel disc $U$ of $p$ of period $n$. Since $\left.p^{n}\right|_{U}$ is conjugate to an irrational rotation, there is a sequence $n_{k}$ such that $\left.p^{n_{k}}\right|_{U} \rightarrow \mathrm{id}$

Set $w_{k}:=\left(\left.p^{n_{k}}\right|_{U}\right)^{-1}(w)$; then $w_{k} \rightarrow w$. Fix $\zeta_{0} \in f^{-1}(w)$ and let $D$ be a neighbourhood of $\zeta_{0}$ on which $f$ is injective. By disregarding finitely many entries, we 
can ensure that $w_{k} \in f(D)$ for all $k \geq 1$. Let us define $\zeta_{k}:=\left(\left.f\right|_{D}\right)^{-1}\left(w_{k}\right)$ and $z_{k}:=\lambda^{n_{k}} \cdot \zeta_{k}$; we may assume that $\left|z_{k}\right| \geq 1$ for all $k$. Then $f\left(z_{k}\right)=w$ and

$$
z_{k} \cdot f^{\prime}\left(z_{k}\right)=\zeta_{k} \cdot f^{\prime}\left(\zeta_{k}\right) \cdot\left(p^{n_{k}}\right)^{\prime}\left(w_{k}\right) \rightarrow \zeta_{0} \cdot f^{\prime}\left(\zeta_{0}\right)
$$

Thus

$$
\sum_{z \in f^{-1}(w) \backslash \mathbf{D}} \frac{1}{|z|^{2}\left|f^{\prime}(z)\right|^{2}} \geq \sum_{k=1}^{\infty} \frac{1}{\left|z_{k}\right|^{2}\left|f^{\prime}\left(z_{k}\right)\right|^{2}}=\infty
$$

as required.

We conclude the section by including a counterexample to Conjecture 1.6.

4.7. Theorem. (Poincaré functions of postcritically finite hyperbolic polynomials) Let $p$ be a polynomial such that every critical point of $p$ eventually maps to a superattracting cycle. Let $z_{0}$ be a fixed point of $p$ that does not belong to the boundary of an invariant Fatou component, and let $f \in \mathcal{S}$ be the corresponding Poincaré function. Then $f$ has no asymptotic values, but nonetheless satisfies (1.2).

Remark. As an example, one can take $p(z)=z^{2}-1$ and $z_{0}=(1+\sqrt{5}) / 2$. This Poincaré function was previously considered in [Mih12, Appendix B] as an example of a function $f \in \mathcal{S}$ having no asymptotic values but critical points of arbitrarily high order.

Proof. By [MP12, Corollary 4.4], the function $f$ has no asymptotic values. Since the filled Julia set $K:=K(p)$ has non-empty interior, it has positive area. Now $f^{-1}(K)$ is completely invariant under multiplication by the multiplier $\lambda$ of $z_{0}$, by the functional equation (3.1). Hence, for all $n \geq 1$,

$$
\int_{z \in \mathbf{C}: 1 \leq|z| \leq|\lambda|^{n} \text { and } f(z) \in K} \frac{\mathrm{d} x \mathrm{~d} y}{|z|^{2}}=n \cdot \int_{z \in \mathbf{C}: 1 \leq|z| \leq|\lambda| \text { and } f(z) \in K} \frac{\mathrm{d} x \mathrm{~d} y}{|z|^{2}}=: \varepsilon \cdot n,
$$

with $\varepsilon>0$. Hence (1.2) holds, as required.

\section{A counterexample to the area property near infinity}

Proof of Theorem 1.8. We shall now show that there exists an entire function $f \in \mathcal{B}$ that violates the area property near infinity, using a construction from [Rem14, Section 7]. As indicated in the introduction, and stated in [Rem14] (following Theorem 1.11 in that paper), it can be shown that the exact function considered in that paper, which is a hyperbolic entire function with full hyperbolic dimension, also violates the area property. However, it shall be slightly more convenient for us to use the same construction, but with different parameters. We discuss the original example following the completion of the proof.

As in [Rem14], the proof proceeds in two steps:

- First, a simply-connected domain $V$ is constructed that does not intersect its $2 \pi i$-translates, along with a conformal isomorphism

$$
G: V \rightarrow H:=\left\{x+i y: x>-14 \log _{+}|y|\right\},
$$

where again $\log _{+}|y|=\max (0, \log |y|)$. The tract is chosen such that the (not globally defined) function $g: \exp (V) \rightarrow \mathbf{C} ; g(\exp (z))=\exp (G(z))$ does not have the area property near infinity. 
- By [Rem14, Theorem 1.7], the function $g$ can be approximated by a transcendental entire function $f \in \mathcal{B}$ with $|f(z)-g(z)|=O(1 /|z|)$ for $z \in \exp (V)$, and $|f(z)|=O(1 /|z|)$ elsewhere. It can then be checked that $f$ also does not have the area property near infinity.

For the first step, we shall use [Rem14, Lemma 7.2 and Theorem 7.4], which imply that the domain $V$ and the function $G$ can be chosen such that the following properties hold.

(1) $V \subsetneq\{a+i b: a>1$ and $|b|<\pi\}$;

(2) there are $C_{1}>1$ and $Q \geq 1$ such that $\operatorname{Re} z / C_{1} \leq \log _{+}|G(z)| \leq C_{1} \operatorname{Re} z$ for all $z \in V$ with $\operatorname{Re} z \geq Q$.

(3) there exist constants $C_{2}>0, k_{0} \geq Q / 2 \pi$ and a collection of points $\left(\zeta_{k}\right)_{k \geq k_{0}}$ in $V$ such that $\operatorname{Re} \zeta_{k}=2 \pi k$, $\operatorname{dist}\left(\zeta_{k}, \partial V\right) \geq C_{2}$ and $\operatorname{Re} G\left(\zeta_{k}\right)=1$ for all $k \geq k_{0}$.

We remark that $V$ consists of a central strip of fixed width, to which a sequence of "side chambers" are attached; see [Rem14, Figure 1]. These are equally spaced in a $2 \pi$-periodic manner, and the $k$-th chamber is connected to the central strip by a small opening of size $\varepsilon_{k}$. The points $\zeta_{k}$ are precisely the mid-points of these chambers, and the opening size $\varepsilon_{k}$ is chosen such that $\operatorname{Re} G\left(\zeta_{k}\right)=1$. This ensures property (3). Property (2) is a simple consequence of the description of the tract and classical geometric function theory. For details, we refer to [Rem14, Section 7].

Let us verify that, when $G$ is chosen with properties (1) to (3), we have

$$
\sum_{m \in \mathbf{Z}}\left|\left(G^{-1}\right)^{\prime}(1+2 \pi i m)\right|^{2}=\infty
$$

As in Proposition 4.3, the formula (5.1) implies via Koebe's Distortion Theorem that $g$ does not have the area property near infinity. (By this, we mean that $g^{-1}(K)$ has infinite cylindrical area for any compact set $K \subset \mathbf{C} \backslash \overline{\mathbf{D}}$ with nonempty interior.)

Claim 1. There is a constant $C_{3}>1$ such that $k / C_{3} \leq \operatorname{dist}\left(G\left(\zeta_{k}\right), \partial H\right) \leq C_{3} \cdot k$ for all $k \geq k_{0}$.

Proof. By (3) above, we have $\operatorname{Re} \zeta_{k}=2 \pi k \geq Q$, and hence $\log _{+}|G(z)| \leq 2 \pi C_{1} k$ by (2). On the other hand, $\operatorname{dist}(\zeta, \partial H) \leq 1+14 \log _{+} \operatorname{Im} \zeta \leq 15 \log _{+}|\zeta|$ whenever $\zeta \in H$ with $\operatorname{Re} \zeta=1$. This implies the upper bound; the lower bound follows analogously.

Since $G$ is a conformal isomorphism, we see from the standard estimate (1.3), together with Claim 1 and (3), that

$$
\left|G^{\prime}\left(\zeta_{k}\right)\right|=\frac{\rho_{V}\left(\zeta_{k}\right)}{\rho_{H}\left(G\left(\zeta_{k}\right)\right)} \leq 4 \cdot \frac{\operatorname{dist}\left(G\left(\zeta_{k}\right), \partial H\right)}{\operatorname{dist}\left(\zeta_{k}, \partial V\right)} \leq \text { const } \cdot k .
$$

Now, for $k \geq k_{0}$, consider the set $\mathcal{M}_{k}:=\left\{m \in \mathbf{N}:\left|2 \pi m-\operatorname{Im} G\left(\zeta_{k}\right)\right| \leq k /\left(5 C_{3}\right)\right\}$. By (5.2) and Koebe's Distortion Theorem, we have

$$
\mid\left(G^{-1}\right)^{\prime}(1+2 \pi i m) \geq \frac{\text { const }}{k}
$$

when $m \in \mathcal{M}_{k}$. Also note that

$$
\# \mathcal{M}_{k} \geq\left\lfloor\frac{k}{5 \pi C_{3}}\right\rfloor \geq \text { const } \cdot k
$$

for all $k \geq k_{1}:=\max \left(\left\lceil 5 \pi C_{3}\right\rceil, k_{0}\right)$ (for a constant independent of $k$ ). 
Claim 2. The sets $\mathcal{M}_{k}$ are pairwise disjoint.

Proof. Consider the vertical line segment $S$ in $H$ from $G\left(\zeta_{k}\right)-k i /\left(5 C_{3}\right)$ to $G\left(\zeta_{k}\right)+k i /\left(5 C_{3}\right)$. By Claim 1, we have

$$
\operatorname{dist}(\zeta, \partial H) \geq \frac{4 k}{5 C_{3}}
$$

for all $\zeta \in S$. By the standard estimate (1.3), we see that the hyperbolic distance in $H$ between the midpoint $G\left(\zeta_{k}\right)$ and any point of $S$ is at most $1 / 2$. Recall that $1+2 \pi i m \in S$ for all $m \in \mathcal{M}_{k}$, by definition.

On the other hand, the hyperbolic distance in $V$ between $\zeta_{k}$ and $\zeta_{k^{\prime}}$, with $k \neq k^{\prime}$, is strictly larger than 1 . Indeed, the hyperbolic distance between $\zeta_{k}$ and $\zeta_{k^{\prime}}$ in the strip $\{a+i b:|a|<\pi\} \supset V$ is strictly larger than one by direct computation, and the claim follows from the comparison principle of hyperbolic geometry.

Combining all the estimates, we see that

$$
\begin{aligned}
\sum_{m \in \mathbf{Z}}\left|\left(G^{-1}\right)^{\prime}(1+2 \pi i m)\right|^{2} & \geq \sum_{k=k_{1}}^{\infty} \sum_{m \in \mathcal{M}_{k}}\left|\left(G^{-1}\right)^{\prime}(1+2 \pi i m)\right|^{2} \\
& \geq \text { const } \cdot \sum_{k=k_{1}}^{\infty} \# \mathcal{M}_{k} \cdot \frac{1}{k^{2}} \geq \text { const } \cdot \sum_{k=k_{1}}^{\infty} \frac{1}{k}=\infty
\end{aligned}
$$

This establishes (5.1).

Now let $f \in \mathcal{B}$ be the entire function satisfying $|f(z)-g(z)|=O(1 /|z|)$ for $z \in \exp (V)$, whose existence is guaranteed by [Rem14, Theorem 1.7]. It is not difficult to verify, using the above information about the construction, that $f$ does not have the area property near infinity. Instead, we shall derive this fact using quasiconformal equivalence. By [Rem14, Theorem 1.8], there is $R>0$ and a quasiconformal map $\varphi: \mathbf{C} \rightarrow \mathbf{C}$ such that $g(\varphi(z))=f(z)$ whenever $|f(z)|>R$. Moreover, according to [Rem14, Theorem 6.3], the map $\varphi$ can be chosen such that $\varphi(z)=z$ on $f^{-1}(D)$, where $D$ is a simply-connected neighbourhood of $S(f)$.

In particular, the dilatation of $\varphi$ is supported on the set

$$
\{z \in \mathbf{C}:|f(z)| \leq R \text { and } f(z) \notin D\} .
$$

If $f$ had the area property near $\infty$, then this set would have finite cylindrical area, and hence $\varphi$ would preserve the property of having finite cylindrical area by Proposition 4.2. But then $g$ also has the area property (due to the functional relation $g(\varphi(z))=f(z))$, which is a contradiction.

Remark. The function $G$ constructed in [Rem14, Section 7] has the same properties as above, except that, in (3), the points $\zeta_{k}$ satisfy $\operatorname{Re} G\left(\zeta_{k}\right) \asymp k$, rather than $\operatorname{Re} G\left(\zeta_{k}\right)=1$. (This is what enables the construction of a suitable iterated function system, which gives rise to the desired hyperbolic sets.) Moreover, this is not required to hold for all sufficiently large $k$ (although that can be arranged), but only for those satisfying $K_{i}<2 \pi k+R_{0}<3 K_{i}$ for some $i$, where $\left(K_{i}\right)$ is a (possibly rapidly) increasing sequence and $R_{0}$ is a universal constant; see [Rem14, Corollary 7.5]. Without loss of generality, let us assume that $K_{0} \geq \max \left(2 \pi, R_{0}\right)$.

To see that this function also satisfies (5.1), and hence violates the area property near infinity, define a sequence $\left(\tilde{\zeta}_{k}\right)$ by $\tilde{\zeta}_{k}:=G^{-1}\left(1+i \cdot \operatorname{Im} G\left(\zeta_{k}\right)\right)$. By $(2)$, we see that $\log _{+} \operatorname{Im} G\left(\zeta_{k}\right) \geq$ const $\cdot k$, and hence the hyperbolic distance (in $H$ ) between 
$\zeta_{k}$ and $\tilde{\zeta}_{k}$ is bounded from above, independently of $k$. Thus $\operatorname{dist}\left(\tilde{\zeta}_{k}, \partial V\right) \geq$ const, independently of $k$, and we can apply the same argument as above, replacing $\zeta_{k}$ by $\tilde{\zeta}_{k}$. We conclude that indeed

$$
\begin{aligned}
\sum_{m \in \mathbf{Z}}\left|\left(G^{-1}\right)^{\prime}(1+2 \pi i m)\right|^{2} & \geq \sum_{i \geq 0} \sum_{k=\left\lfloor\left(K_{i}-R_{0}\right) / 2 \pi\right\rfloor+1}^{\left\lceil\left(3 K_{i}-R_{0}\right) / 2 \pi\right\rceil-1} \frac{\text { const }}{k} \\
& \geq \text { const } \cdot \sum_{i \geq 0}\left\lfloor\frac{2 K_{i}}{2 \pi}\right\rfloor \cdot \frac{1}{3 K_{i}-R_{0}} \geq \text { const } \cdot \sum_{i \geq 0} \frac{K_{i}}{2 K_{i}}=\infty .
\end{aligned}
$$

\section{Quadratic differentials}

In this section, we discuss a matter that is closely connected to the area property, namely the behaviour of quadratic differentials under the pushforward by an entire function. Recall that a quadratic differential is a tensor of the form $q(z) \mathrm{d} z^{2}$ (in local coordinates). If the local coefficient $q$ can always be chosen to be measurable, holomorphic or meromorphic, then the differential itself is called measurable, holomorphic or meromorphic. A pole of a meromorphic quadratic differential is then a point near which the local coefficient must have a pole; observe that locally such a differential can always be written in the form $\mathrm{d} z^{2} / z^{d}$, where $d$ is the order of the pole. Any quadratic differential gives rise to an associated area form $|q(z)|\left|\mathrm{d} z^{2}\right|$; the total area

$$
\int|q(z)|\left|\mathrm{d} z^{2}\right|
$$

is referred to as the (total) mass of the differential. By an elementary calculation, the mass of a meromorphic quadratic differential is finite near a simple pole, but infinite near a pole of higher order.

Quadratic differentials play a key role in complex dynamics and complex analysis. Of particular interest is the pushforward operator: If $f: U \rightarrow V$ is an analytic function and $q=q(z) \mathrm{d} z^{2}$ is a measurable (in the following usually meromorphic or even holomorphic) quadratic differential, then the pushforward of $q$ is the formal sum

$$
f_{*} q:=\left(\sum_{f(z)=w} \frac{q(z)}{f^{\prime}(z)^{2}}\right) \mathrm{d} w^{2} .
$$

Of course, in general this sum may or may not converge; if it converges absolutely, we shall say that $q$ is $f$-summable.

If $q$ is a meromorphic quadratic differential on the Riemann sphere with at most finite poles, then $q$ has finite total mass. Since the pushforward under a holomorphic map can never increase mass (and may in fact decrease it due to possible cancellations), it follows that such a differential is always summable. On the other hand, for quadratic differentials with at worst double poles the total mass is infinite, so this argument cannot be used to show that the differential is $f$-summable. (Quadratic differentials with double poles can play an important role in holomorphic dynamics; for example, they appear when bounding the number of non-repelling cycles, see [Eps99].) However, if $f$ is entire and has the area property, then the pushforward converges absolutely at least for $w \notin S(f)$. If furthermore $f \in \mathcal{B}$, then we can say more about the nature of the singularity of this pushforward near $\infty$. This is a consequence of the following observation. 
6.1. Proposition. (Quadratic differentials and logarithmic singularities) Let $V \subsetneq \mathbf{C}$ be an unbounded simply-connected domain, and let $f: V \rightarrow \mathbf{C} \backslash \overline{\mathbf{D}}=: W$ be a holomorphic universal covering map. Suppose furthermore that

$$
\sum_{z \in f^{-1}(w) \backslash \mathbf{D}} \frac{1}{|z|^{2} \cdot\left|f^{\prime}(z)\right|^{2}}<\infty
$$

for all $w \in W$. Let $q=q(z) \mathrm{d} z^{2}$ be a holomorphic quadratic differential on $V$ for which there is a constant $C>0$ such that $|q(z)| \leq C /|z|^{2}$ for all sufficiently large $z \in V$. Then $q$ is $f$-summable for all $w \in W$ and the pushforward $f_{*} q$ has at most a double pole at $\infty$.

Proof. For convenience, we may assume that $0 \notin \bar{V}$ (restricting $V$ to the complement of a slightly larger disc and reparametrizing, if necessary). This ensures that we can write $q=\left(\rho(z) / z^{2}\right) \mathrm{d} z^{2}$ for all $z \in \mathcal{V}$, with $\rho(z) \leq \tilde{C}$ for a suitable constant $\tilde{C}>0$. The pushforward is then the formal sum

$$
f_{*} q=\left(\sum_{z \in f^{-1}(w)} \frac{\rho(z)}{f^{\prime}(z)^{2} z^{2}}\right) \mathrm{d} w^{2}=: \sigma(w) \mathrm{d} w^{2} .
$$

By virtue of the area property (6.1) for $f$, the sum is absolutely convergent for $w \in W$, so $\sigma$ is defined and holomorphic on $W$. (Recall that the sum (6.1) converges locally uniformly in $w$ due to the Koebe Distortion Theorem.) We must show that

$$
|\sigma(w)|=o(1 /|w|) \quad \text { as } w \rightarrow 0 .
$$

To begin, let us perform a logarithmic change of variable in the sense of Eremenko and Lyubich [EL92] as follows. Let $U$ be a connected component of $\exp ^{-1}(V)$. Since $f \circ \exp : U \rightarrow W$ is a universal covering map, there is a conformal isomorphism $\varphi$ from $\mathbf{H}:=\{a+i b: a>0\}$ to $U$ such that $f(\exp (\varphi(\zeta)))=\exp (\zeta)$ for all $\zeta \in \mathbf{H}$.

Let $\zeta \in \mathbf{H}$ with $\operatorname{Re} \zeta>1$, and define $\tilde{\zeta}:=1+i \operatorname{Im} \zeta$. By Koebe's Distortion Theorem, applied to the restriction of $\varphi$ to the disc of radius $\operatorname{Re} \zeta$ around $\zeta$, we see that

$$
\left|\varphi^{\prime}(\zeta)\right| \leq 8 \operatorname{Re} \zeta \cdot\left|\varphi^{\prime}(\tilde{\zeta})\right|
$$

Now let $w \in W$ with $|w|>e$, and let $\zeta_{0} \in \exp ^{-1}(w)$. We set $\tilde{w}:=e \cdot w /|w|$, so that $|\tilde{w}|=e$. Differentiating the relation $f \circ \exp \circ \varphi=\exp$, we see that

$$
f^{\prime}(z) \cdot z=\frac{e^{\zeta}}{\varphi^{\prime}(\zeta)}
$$

whenever $z=e^{\varphi(\zeta)}$. Hence

$$
\begin{aligned}
|\sigma(x)| & \leq \sum_{z \in f^{-1}(w)} \frac{|\rho(z)|}{|z|^{2} \cdot\left|f^{\prime}(z)\right|^{2}} \leq \frac{\tilde{C}}{|w|^{2}} \cdot \sum_{m \in \mathbf{Z}}\left|\varphi^{\prime}\left(\zeta_{0}+2 \pi i m\right)\right|^{2} \\
& \leq \frac{8 \tilde{C}\left(\operatorname{Re} \zeta_{0}\right)^{2}}{|w|^{2}} \cdot \sum_{m \in \mathbf{Z}}\left|\varphi^{\prime}\left(1+\left(2 \pi m+\operatorname{Im} \zeta_{0}\right) i\right)\right|^{2} \\
& =\frac{8 \tilde{C}(\log |w|)^{2}}{|w|^{2}} \cdot \sum_{z \in f^{-1}(\tilde{w})} \frac{e^{2}}{|z|^{2} \cdot\left|f^{\prime}(z)\right|^{2}}
\end{aligned}
$$




$$
\leq \text { const } \cdot \frac{(\log |w|)^{2}}{|w|^{2}} \max _{|\omega|=e} \sum_{z \in f^{-1}(\omega)} \frac{1}{|z|^{2} \cdot\left|f^{\prime}(z)\right|^{2}} .
$$

The maximum on the right-hand side is finite by (6.1). So indeed

$$
|\sigma(x)| \leq \mathrm{const} \cdot \frac{(\log |w|)^{2}}{|w|^{2}}=o(1 /|w|) \quad \text { as } w \rightarrow \infty .
$$

We can deduce the following global statement.

6.2. Corollary. (Pushforwards of QD under class $\mathcal{S}$ maps with the area property) Let $f \in \mathcal{S}$ have the area property, and let $q$ be a meromorphic quadratic differential on $\hat{\mathbf{C}}$, with at most double poles. Then $f_{*} q$ is also a meromorphic quadratic differential on $\hat{\mathbf{C}}$ with at most double poles. More precisely, $f_{*} q$

- has at most double poles at $\infty$, asymptotic values of $f$, and at the images of double poles of $q$;

- has at most simple poles at non-asymptotic critical values of $f$ and at the images of simple poles of $q$;

- is holomorphic elsewhere.

Proof. Let us again write $\rho(z)=\rho(z) \frac{\mathrm{d} z^{2}}{z^{2}}$, where $\rho$ is meromorphic on $\hat{\mathbf{C}}$ and satisfies $\rho(z)=O(1)$ as $w \rightarrow \infty$. Then

$$
f_{*} q=\left(\sum_{z \in f^{-1}(w)} \frac{\rho(z)}{f^{\prime}(z)^{2} z^{2}}\right) \mathrm{d} w^{2}=: \sigma(w) \mathrm{d} w^{2}
$$

is defined and holomorphic by the area property, except possibly at singular values of $f$ (including $\infty$ ) and the images of poles of $q$. Let $E$ denote this finite exceptional set; we must investigate the behaviour of $\sigma$ near a point $w_{0} \in E$.

By postcomposing with a Möbius transformation, we may assume that $w_{0}=0$ and that $E \cap \mathbf{D}=\{0\}$. Let $\mathcal{V}$ denote the set of all connected components of $f^{-1}(\mathbf{D})$. For every $V \in \mathcal{V}$, denote the pushforward of $q$ under the restriction $\left.f\right|_{V}$ by $\sigma_{V}(w) \mathrm{d} w^{2}$. Then $\sigma$ is defined and holomorphic on the punctured disc $\mathbf{D}^{*}$.

(a) If $f: V \rightarrow \mathbf{D}^{*}$ is a universal covering, then $\sigma_{V}$ has at most a double pole at 0 by Proposition 6.1 (applied to $1 / f$ ).

(b) Otherwise, $f: V \backslash f^{-1}(0) \rightarrow \mathbf{D}^{*}$ is a finite-degree covering map, and it follows that $V$ contains exactly one element $z_{0}$ of $f^{-1}(0)$, and that $f: V \rightarrow \mathbf{D}$ is a branched covering map with no critical points except possibly at $z_{0}$. The local pushforward of a meromorphic quadratic differential defined near a critical point is well-understood (and can be verified by a simple computation in local coordinates); see [Eps99, Formula (4)]. In particular, it has the following properties.

- The local pushforward of a quadratic differential with at most double poles under a holomorphic map has at most a double pole; hence $\sigma_{V}$ has at most a double pole at 0 .

- If $q$ has at most a simple pole at $z_{0}$, then $\sigma_{V}$ has at most a simple pole at 0. (This also follows by considering the mass of $q$, as mentioned above.)

- If $q$ is holomorphic at $z_{0}$ and $f: V \rightarrow \mathbf{D}$ is a conformal isomorphism, then clearly $f_{*} q$ is holomorphic at 0 . 
So we have seen that each $\sigma_{V}$ has at most a double pole at zero. Recall that

$$
\sigma(w)=\sum_{V \in \mathcal{V}} \sigma_{V}(w)
$$

on $\mathbf{D}^{*}$, where the sum converges locally uniformly. It follows (e.g. by comparing the Laurent series of $\sigma(w)$ with that of the partial sums) that $\sigma$ also has at most a double pole at zero. The claim about simple poles follows analogously.

It is interesting to consider when $f_{*} q$ acquires at most simple poles also at finite asymptotic values or at infinity. For example, set $f(z):=e^{z}$ and $q:=\mathrm{d} z^{2} / z^{2}$. We have

$$
f_{*} q=\sum_{k=-\infty}^{\infty} \frac{\mathrm{d} w^{2}}{w^{2}(\log w+2 \pi i k)^{2}}=\frac{-\pi \mathrm{d} w^{2}}{4 w^{2} \sin ^{2} \frac{\log w}{2 i}}=\frac{\pi \mathrm{d} w^{2}}{w^{3}-2 w^{2}+w}
$$

(where we used the infinite partial fraction expansion of $\frac{1}{\sin ^{2}}$.) Hence $f_{*} q$ indeed has only a simple pole at $\infty$. This phenomenon means that the pushforward results in a massive cancellation of mass near infinity.

More generally, consider the following strengthening of the area property:

$$
\sum_{z \in f^{-1}(w) \backslash \mathbf{D}} \frac{1}{|z|^{t}\left|f^{\prime}(z)\right|^{t}}<\infty
$$

for some $t=2-\varepsilon<2$. A simple estimate shows that, for $f(z)=\exp (z)$, this property is satisfied for all $t>1$. Furthermore, if $f$ is a Poincaré function for a postcritically finite polynomial $p$, then (6.4) holds for some $t<2$. Indeed, as in inequality (4.4) in the proof of Theorem 4.6, we see that the corresponding series is bounded by the Poincaré series for $p$ with exponent $t$. This series converges when $t>\delta(p)$, where $\delta(p)$ is the critical exponent for the Poincare series. For postcritically finite maps, it is known that $\delta(p)<2$ coincides with the Hausdorff dimension of the Julia set (compare [Prz99]).

6.3. Theorem. (Simple poles at asymptotic values) Let $f \in \mathcal{S}$ satisfy (6.4) for some $t<2$, and let $q$ be a meromorphic quadratic differential on the Riemann sphere with at most double poles. Then $f_{*} q$ is a meromorphic quadratic differential on the Riemann sphere with at most double poles at the images of the finite double poles of $q$, and at most simple poles elsewhere.

The theorem follows by replacing Proposition 6.1 in the proof of Corollary 6.2 with the following observation.

6.4. Proposition. (Quadratic differentials and logarithmic singularities, II) Suppose that $f$ and $q$ are as in Proposition 6.1, but that $f$ additionally satisfies (6.4) for some $t<2$. Then $f_{*} q$ has at most a simple pole at 0 .

Proof. By a well-known estimate of Eremenko and Lyubich [EL92, Lemma 1], we have

$$
\left|z \cdot f^{\prime}(z)\right| \geq \text { const } \cdot|f(z)| \cdot|\log | f(z)||
$$

for such a universal covering when $|f(z)|$ is sufficiently large. Hence,

$$
\sum_{z \in f^{-1}(w) \backslash \mathbf{D}} \frac{1}{|z|^{2}\left|f^{\prime}(z)\right|^{2}} \leq \frac{1}{|w|^{\varepsilon}} \cdot \sum_{z \in f^{-1}(w) \backslash \mathbf{D}} \frac{1}{|z|^{2-\varepsilon}\left|f^{\prime}(z)\right|^{2-\varepsilon}},
$$


provided that $\varepsilon$ is chosen such that the sum on the right converges. (This is possible by (6.4).) In particular, if we replace the exponent 2 by the exponent $2-\varepsilon$ in the estimate (6.2) in the proof of Proposition 6.1, we obtain

$$
|\sigma(x)| \leq O\left((\log |w|)^{2} /|w|^{2+\varepsilon}\right)=o\left(1 /|w|^{2}\right),
$$

showing that $f_{*} q$ has at most a simple pole.

Remark 1. Let $f$ be the Poincaré function of a postcritically finite polynomial (or rational map) $p$ at a repelling periodic point. Let $q=\mathrm{d} z^{2} / z^{2}$; then $q$ is invariant under the map $\lambda$ (multiplication by the multiplier of the repelling cycle). Using the functional relation (3.1), we see that

$$
p_{*} f_{*} q=f_{*} \lambda_{*} q=f_{*} q,
$$

so $f_{*} q$ is pushforward invariant under $p$. (Note that the pushforward considered in (6.3) is precisely of this type, using $f(z)=\exp (z)$ and $p(z)=z^{2}$.)

We observed in Theorem 6.3 that $f_{*} q$ has at most a simple pole at infinity (and at most double poles elsewhere). The set of non-trivial quadratic differentials that are pushforward invariant under a postcritically finite polynomial or rational map is an intriguing object. Unless the rational map in question is a Lattès example, pushing forward a quadratic differential with at most simple poles eventually results in some cancellation of mass. (This is infinitesimal Thurston contraction [DH93, Lemma 1 on p. 272]; compare also [Eps99, Lemma 3].) Hence a push-forward invariant quadratic differential must have at least a double pole somewhere. Some quadratic differentials of this type can be obtained from algebraic expressions between multipliers of repelling cycles; the above construction yields another set of examples. It seems interesting to study their properties.

Remark 2. Suppose that $f \in \mathcal{B}$ satisfies the area property (or its stronger variation (6.4)). If $q$ is a meromorphic quadratic differential with at most double pole at $\infty$, then Propositions 6.1 and 6.4 still imply that $f_{*} q$ is defined for sufficiently large values of $w$ and has at most a double (resp. simple) pole at $\infty$. However, there is no reason to expect the pushforward to be globally meromorphic.

\section{Further comments and questions}

Meromorphic functions. We have stated our theorems, for the most part, for entire rather than meromorphic functions, as it is known classically that invariance of order can fail in the latter case. However, essentially all our results also apply, with the same proof, to meromorphic functions. (We remark that the area property was defined for entire functions, but it extends verbatim to the meromorphic case.) In particular, Corollary 6.2 and Theorem 6.3 extend to meromorphic functions with finite singular sets, using exactly the same proof.

An exception is given by Theorem 4.6, concerning Poincaré functions of polynomials. In the proof of this theorem, we used the fact that $p$ has nonempty Fatou set, but did not otherwise rely on the fact that $p$ is a polynomial. Hence Theorem 4.6 holds also for a Poincaré function $f$ of a transcendental entire function $h$ or of a rational function $h$, provided that the Fatou set of $h$ is non-empty. However, in the case where $F(h)=\emptyset$, it is possible for the area property to fail. Indeed, consider the case where $h$ is a Lattès map; that is, a (postcritically finite) rational function obtained from a linear toral endomorphism via projection to the Riemann sphere. 
By definition, the linearizer $f$ is an elliptic function, namely the projection from the torus in question to the sphere. Any doubly-periodic set of positive area has infinite cylindrical area, since $\sum_{\lambda \in \Lambda \backslash\{0\}} 1 /|\lambda|^{2}=\infty$ for any lattice $\Lambda \subset \mathbf{C}$. Hence $f$ does not have the area property.

The general answer turns out to depend on the measurable dynamics of the function $h$. Indeed, it is well-known that, for a Lattès map, almost every orbit is dense in the Riemann sphere. As the following result, which extends Theorem 4.6, shows, this is what causes the failure of the area property.

7.1. Theorem. (Poincaré functions of rational or transcendental entire functions) Suppose that the transcendental meromorphic function $f$ is a Poincare function of some entire or rational function $h$. If $F(h) \neq \emptyset$, then $f$ has the area property if and only if $h$ has no rotation domains. Otherwise, $f$ has the area property if and only if $\operatorname{dist}\left(h^{n}(z), \mathcal{P}(h)\right) \rightarrow 0$ for almost all $z \in \mathbf{C}$.

Sketch of proof. By the above remarks, it suffices to consider the case where $F(h)$ is empty. As in the proof of Theorem 4.6, and as remarked after (6.4), the area property is equivalent to the question whether the Poincare series of $h$ converges for the exponent 2 at $w \notin \mathcal{P}(h)$. The fact that this is the case if and only if $\operatorname{dist}\left(h^{n}(z), \mathcal{P}(h)\right) \rightarrow 0$ is surely known, at least for rational functions; for completeness, we sketch a proof below.

If $\mathcal{P}(h)=\mathbf{C}$, then there is nothing to prove (since the area property only makes statements about sets disjoint from the singular set of $f$, it holds trivially). Otherwise, let $w \in \mathbf{C} \backslash \mathcal{P}(h)$. By the existence of nice sets (proved by Rivera in the rational case and Dobbs [Dob11] in the general case), there is a small simply-connected open set $U \subset \mathbf{C} \backslash \mathcal{P}(h)$ around $w$, such that $h^{n}(\partial U) \cap \partial U=\emptyset$ for all $n \geq 1$. The pullbacks of $U$ along first returns to $U$ form an infinite conformal iterated function system (IFS). It is easy to see that the Poincare series for $f$ corresponding to exponent 2 will converge if and only if the sum, over all levels $n$, of the total area of the sets of level $n$ in this IFS converges. Here by a "set of level $n$ ", we mean the result of applying a composition of $n$ of the contractions defining the IFS. In other words, the sets of level $n$ are precisely the domains of the $n$-th return map to $U$.

If $\operatorname{dist}\left(h^{n}(z), \mathcal{P}(h)\right) \rightarrow 0$ for almost all $z \in \mathbf{C}$, then there is a positive measure set of points in $U$ that never return to $U$ under iteration. It follows that the areas in question decrease geometrically, and hence the Poincaré series converges. On the other hand, if there is a positive measure set of points with $\lim \sup \operatorname{dist}\left(h^{n}(z), \mathcal{P}(h)\right) \not \rightarrow 0$, then by a well-known argument (see e.g. [RVS11, Theorem 3.3]) almost every orbit is dense. In particular, the level $n$ sets of the IFS have full area in $U$, and hence the Poincaré series diverges.

The area property and measurable dynamics. In several places, our work suggests close connections between the area property and measurable dynamics. One such connection concerns the case of Poincaré functions, where we have seen that the measurable dynamics of the original map (here: the Poincaré series), are reflected in the value distribution (here: the area property and its generalizations) for the linearizer. We remark that Eremenko and Sodin [ES90] used this type of connection to give a new proof of the uniqueness of measures of maximal entropy for rational functions. 
Perhaps more interestingly, it appears that there are connections between conditions such as the area property and its stronger variant (6.4) and the measurable dynamics of the transcendental function itself. Indeed, we already saw that the same construction that leads to a hyperbolic entire function with full hyperbolic dimension also yields a function for which the area property fails. Furthermore, such connections are suggested by work of Urbański with several collaborators (see e.g. [UZ03, MU08]) on the existence of conformal and invariant measures, and Hausdorff dimension of radial Julia sets, for finite-order entire and meromorphic functions.

In particular, in [MU08], a class of hyperbolic meromorphic functions of finite order is treated that satisfy a strong regularity of growth property, known there as the balanced condition. In the case of a finite-order entire function $f \in \mathcal{B}$, the function is balanced if and only if

$$
\left|f^{\prime}(z)\right| \asymp(1+|z|)^{\rho(f)-1} \cdot(1+|f(z)|)
$$

for all $z \in J(f)$ [MU08, Lemma 3.1]. It follows from this condition that (6.4) holds for all $t>1$, and hence all of these functions have the area property and satisfy the conclusions of Theorem 6.3. In particular, this implies that Poincaré functions of postcritically finite polynomials with non-smooth Julia sets are not balanced in the sense of Mayer and Urbański. Indeed, the critical exponent $c$ of the Poincaré series of such a polynomial coincides with the Hausdorff dimension of its Julia set, which is larger than one. It follows that the Poincaré function does not satisfy (6.4) for $1<t<c$.

It is plausible that the minimal exponent in (6.4) is connected to the concept of eventual hyperbolic dimension, which is defined in analogy with [RS10, Section 5] as follows:

$$
\operatorname{edim}_{\text {hyp }}(f):=\lim _{R \rightarrow \infty} \sup \left\{\operatorname{dim} K: K \text { hyperbolic, } \min _{z \in K}|z| \geq R\right\} .
$$

(Recall that a set is hyperbolic for $f$ if it is compact, invariant and expanding.)

Classes of functions with the area property. Given the many interesting connections between the area property and interesting applications in complex dynamics and function theory, it makes sense to identify classes of entire transcendental functions $f \in \mathcal{B}$ having the area property. As mentioned above, the balanced condition of Mayer and Urbański [MU08] gives rise to such a class of functions, but it is rather restrictive; in particular, it does not include Poincaré functions, which have been our primary source of non-trivial examples. We believe that there should be a natural geometric condition on the tracts of an entire transcendental function $f \in \mathcal{B}$ that covers all balanced functions in this class (in the sense of (7.1)) as well as all Poincare functions of postcritical polynomials. This condition should ensure that the area property holds and that quadratic differentials with at most a double pole push forward to at most a simple pole at infinity. Furthermore, hyperbolic functions in the corresponding quasiconformal equivalence class should have hyperbolic dimension strictly less than two. As this question takes us beyond the scope of this note, it will be left to a subsequent paper.

\section{References}

[Ahl06] Ahlfors, L. V.: Lectures on quasiconformal mappings. Second edition. With supplemental chapters by C. J. Earle, I. Kra, M. Shishikura and J. H. Hubbard. - Univ. Lecture Ser. 38, Amer. Math. Soc., Providence, RI, 2006. 
[Ast94] Astala, K.: Area distortion of quasiconformal mappings. - Acta Math. 173:1, 1994, $37-60$.

[Bea91] Beardon, A. F.: Iteration of rational functions. - Grad. Texts in Math. 132, SpringerVerlag, New York, 1991.

[BM07] Beardon, A.F., and D. Minda: The hyperbolic metric and geometric function theory. - In: Quasiconformal mappings and their applications, Narosa, New Delhi, 2007, 9-56.

[BE95] Bergweiler, W., and A. Eremenko: On the singularities of the inverse to a meromorphic function of finite order. - Rev. Mat. Iberoam. 11:2, 1995, 355-373.

[Bis12] Bishop, C. J.: The order conjecture fails in $\mathcal{S}$. - J. Anal. Math. (to appear).

[Dob11] DobBs, N.: Nice sets and invariant densities in complex dynamics. - Math. Proc. Cambridge Philos. Soc. 150:1, 2011, 157-165.

[DH93] Doundy, A., and J. H. HubBard: A proof of Thurston's topological characterization of rational functions. - Acta Math. 171:2, 1993, 263-297.

[DO08] Drasin, D., and Y. OkuYama: Singularities of Schröder maps and unhyperbolicity of rational functions. - Comput. Methods Funct. Theory 8:1-2, 2008, 285-302.

[Elf34] Elfving, G.: Über eine Klasse von Riemannschen Flächen und ihre Uniformisierung. Acta Soc. Sci. Fenn. 2, 1934.

[Eps99] Epstein, A.: Infinitesimal Thurston rigidity and the Fatou-Shishikura inequality. - Stony Brook IMS Preprint 1999/1, arXiv:math/9902158, 1999.

[Eps02] Epstein, A.: Schwarzian derivatives of topologically finite meromorphic functions. - Ann. Acad. Sci. Fenn. Math. 27:1, 2002, 215-220.

[EL92] Eremenko, A. E., and M. Yu. Lyubich: Dynamical properties of some classes of entire functions. - Ann. Inst. Fourier (Grenoble) 42:4, 1992, 989-1020.

[ES90] Eremenko, A. E., and M. L. Sodin: Iterations of rational functions and the distribution of the values of Poincaré functions. - Teor. Funktsiı̌ Funktsional. Anal. i Prilozhen. 53, 1990, 18-25.

[For81] Forster, O.: Lectures on Riemann surfaces. - Grad. Texts in Math. 81, Springer-Verlag, New York-Berlin, 1981.

[GR66] Gehring, F. W., and E. Reich: Area distortion under quasiconformal mappings. - Ann. Acad. Sci. Fenn. Ser. A I Math. 388, 1966, 15.

[Gey14] Geyer, L.: On the exceptional set in a conditional theorem of Littlewood. - Preprint, arXiv:1404.0983, 2014.

[GO08] Goldberg, A. A., and I. V. OstrovskiI: Value distribution of meromorphic functions. - Transl. Math. Monogr. 236, Amer. Math. Soc., Providence, RI, 2008.

[Hil97] Hille, E.: Ordinary differential equations in the complex domain. - Dover Publications Inc., Mineola, NY, 1997.

[Kün55] KÜNZI, H. P.: Konstruktion Riemannscher Flächen mit vorgegebener Ordnung der erzeugenden Funktionen. - Math. Ann. 128, 1955, 471-474.

[Lan15] LAngley, J. K.: The Schwarzian derivative and the Wiman-Valiron property. - J. Anal. Math. (to appear).

[LV65] Lehto, O., and K. I. Virtanen: Quasikonforme Abbildungen. - Die Grundlehren der mathematischen Wissenschaften in Einzeldarstellungen mit besonderer Berücksichtigung der Anwendungsgebiete, Band, Springer-Verlag, Berlin, 1965.

[MU08] MAYER, V., and M. Urbański: Geometric thermodynamic formalism and real analyticity for meromorphic functions of finite order. - Ergodic Theory Dynam. Systems 28:3, 2008, 915-946. 
[Mih12] Minaljević-Brandt, H.: Semiconjugacies, pinched Cantor bouquets and hyperbolic orbifolds. - Trans. Amer. Math. Soc. 364:8, 2012, 4053-4083.

[MP12] Mihaljević-Brandt, H., and J. Peter: Poincaré functions with spiders' webs. - Proc. Amer. Math. Soc. 140:9, 2012, 3193-3205.

[Nev29] Nevanlinna, F.: Über eine Klasse meromorpher Funktionen. - In: Septième Congrès Math. Scand., 1929, 81-83.

[Nev32] Nevanlinna, R.: Über Riemannsche Flächen mit endlich vielen Windungspunkten. Acta Math. 58:1, 1932, 295-373.

[Nev53] Nevanlinna, R.: Eindeutige analytische Funktionen. 2te Aufl. - Die Grundlehren der mathematischen Wissenschaften in Einzeldarstellungen mit besonderer Berücksichtigung der Anwendungsgebiete, Bd XLVI, Springer-Verlag, Berlin, 1953.

[Pom92] Pommerenke, Ch.: Boundary behaviour of conformal maps. - Grundlehren Math. Wiss. 299, Springer-Verlag, Berlin, 1992.

[Prz99] Przytycki, F.: Conical limit set and Poincaré exponent for iterations of rational functions. - Trans. Amer. Math. Soc. 351:5, 1999, 2081-2099.

[Rem09] Rempe, L.: Rigidity of escaping dynamics for transcendental entire functions. - Acta Math. 203:2, 2009, 235-267.

[RS10] Rempe, L., and G. M. Stallard: Hausdorff dimensions of escaping sets of transcendental entire functions. - Proc. Amer. Math. Soc. 138:5, 2010, 1657-1665.

[RVS11] Rempe, L., and S. VAn Strien: Absence of line fields and Mañé's theorem for nonrecurrent transcendental functions. - Trans. Amer. Math. Soc. 363:1, 2011, 203-228.

[Rem14] Rempe-Gillen, L.: Hyperbolic entire functions with full hyperbolic dimension and approximation by Eremenko-Lyubich functions. - Proc. London Math. Soc. 108:5, 2014, 1193-1225.

[RS99] Rippon, P. J., and G. M. Stallard: Dimensions of Julia sets of meromorphic functions. - J. London Math. Soc. 71:3, 1999, 669-683.

[UZ03] URbański, M., and A. Zdunik: The finer geometry and dynamics of the hyperbolic exponential family. - Michigan Math. J. 51:2, 2003, 227-250.

[Wit68] Wiтtich, H.: Neuere Untersuchungen über eindeutige analytische Funktionen. Zweite, korrigierte Auflage. - Ergeb. Math. Grenzgeb. 8, Springer, 1968. 